\title{
Resurgence in Extended Hydrodynamics
}

\author{
InÊS Aniceto ${ }^{1}$ AND Michą SPALIŃSkI ${ }^{2,3}$ \\ ${ }^{1}$ Institute of Physics, Jagiellonian University, ul. Lojasiewicza 11, 30-348 Kraków, Poland \\ ${ }^{2}$ National Center for Nuclear Research, ul. Hoża 69, 00-681 Warsaw, Poland \\ ${ }^{3}$ Physics Department, University of Biatystok, Konstantego Ciotkowskiego 1L, 15-245 Biatystok, \\ Poland
}

\begin{abstract}
It has recently been understood that the hydrodynamic series generated by the Müller-Israel-Stewart theory is divergent, and that this large-order behaviour is consistent with the theory of resurgence. Furthermore, it was observed that the physical origin of this is the presence of a purely damped nonhydrodynamic mode. It is very interesting to ask whether this picture persists in cases where the spectrum of nonhydrodynamic modes is richer. We take the first step in this direction by considering the simplest hydrodynamic theory which, instead of the purely damped mode, contains a pair of nonhydrodynamic modes of complex conjugate frequencies. This mimics the pattern of black brane quasinormal modes which appear on the gravity side of the AdS/CFT description of $\mathcal{N}=4 \mathrm{SYM}$ plasma. We find that the resulting hydrodynamic series is divergent in a way consistent with resurgence and precisely encodes information about the nonhydrodynamic modes of the theory.
\end{abstract}




\section{Introduction}

Recent years have seen significant advances in the formulation of relativistic hydrodynamic theories [1,2]. This is the result of great interest in the heavy ion collision program, which aims to establish bulk properties of nuclear matter in extreme conditions [3]. Relativistic hydrodynamic models have been essential in uncovering the basic features observed in experiments at RHIC and LHC. As a result, it has become clear that the hydrodynamic approach can be viewed in the same spirit as the effective field theory paradigm of quantum

field theory. This has led to posing (and sometimes answering) foundational questions about the meaning of hydrodynamics.

The point of departure is the idea that the expectation value of the energy-momentum tensor can be expanded in gradients of hydrodynamic variables. It has recently been shown in some specific cases that this hydrodynamic gradient series is divergent [4,5]. Furthermore, the precise way in which the series diverges encodes information about the nonhydrodynamic modes which are not included explicitly in the hydrodynamic description. This pattern is reminiscent of what has been noted in the context of divergent perturbation expansions in other contexts.

The first example of such behaviour of the hydrodynamic gradient expansion was the case of $\mathcal{N}=4$ supersymmetric Yang-Mills theory (SYM), where the series was calculated to high order using the AdS/CFT correspondence [4]. To make the problem manageable, the specific case of boost-invariant flow [6,7] was considered. The gradient expansion was computed up to order 242, and it was observed that the series diverges in factorial fashion. Applying the Borel transform revealed singularities in the Borel plane occurring precisely at the locations corresponding to the complex frequencies of the leading quasinormal modes of the dual black brane geometry. The singularities are related by complex conjugation and are off the real axis, which means that the corresponding degrees of freedom have oscillatory as well as decaying features.

The second example where this kind of behaviour was observed is the hydrodynamic 
series generated by Müller-Israel-Stewart (MIS) theory [8,9]. In this case the series is again divergent, but the singularities in the Borel plane lie on the real axis, which would correspond to purely decaying quasinormal modes. Since in this example one has full control of the problem it is possible to resum the series using ideas from the theory of resurgence [5]. This example is very interesting from the point of view of Borel summation, since the naive application of the inverse Borel transform leads to an imaginary ambiguity. Proper accounting of the nonhydrodynamic degrees of freedom in a way precisely consistent with resurgence theory yields a real and unambiguous result (up to a constant of integration). This result was shown to be consistent with an attractor solution in the original MIS equation, which constitutes a natural definition of the meaning of hydrodynamics beyond the gradient expansion. This gives a strong indication that in cases where a numerical solution is not available, resurgence techniques may be used to mine the hydrodynamic gradient expansion for universal features at times well before hydrodynamic behaviour is typically expected.

The pattern seen in this problem is actually rather typical of the way resurgence theory clarified the role and meaning of perturbative expansions in physics. The role of resurgence in the cancellation of ambiguities in quantum mechanics is well known. In the perturbative study of observables such as the ground state energy of the anharmonic oscillator [10,11, the observable studied was seen to be "non-Borel summable" along the positive real line, since this was a so-called Stokes line with singularities in the corresponding Borel plane. Nevertheless, once all nonperturbative sectors associated with higher multi-instanton corrections were taken into consideration, a process called median resummation was seen to provide a real and unambiguous result (see, e.g., [12 18 for examples of ambiguity cancelation in the context of quantum mechanics 1 and Refs. 21, 22, for its generalisation to quantum field theory). This process consists in the proper summation of all existing

\footnotetext{
${ }^{1}$ It was seen in that in many quantum mechanical systems a very simple exact quantisation condition can be derived for the energy eigenvalues 19,20 , relating perturbative and nonperturbative phenomena, which complements the usual large-order relations coming from resurgence.
} 
sectors (perturbative and nonperturbative) for a given observable, in what is called a transseries. The use of median resummation for the case of transseries with one and two real instanton actions was studied in detail in Ref. [23], and examples of recent applications are Refs. [5] and 24,25].

The cancellation of nonperturbative ambiguities is just one application of a much larger structure behind the asymptotic behaviour of perturbative series. In fact, resurgent analysis and transseries give us a straightforward, systematic path of determining the analytic properties of the observables, the Stokes phenomena associated with singular directions, and the resummation properties leading to unambiguous results..$^{2}$ The crucial role of resurgence in the study of the analytic properties and Stokes phenomena within physical contexts is exemplified in Ref. [44], where from a large- $N$ expansion one can retrieve the properties of the corresponding transseries solution not only for real, finite $N$, but also as an analytic function in the variable $N$ (see also Ref. [45] for another toy example of strong-weak coupling interpolation).

One can ask moreover about the usefulness of the transseries and resurgence in cases where the resummation procedure in the direction of interest is not singular. When our interest is in the result along the positive real line, and the singular directions (Stokes lines) are in the complex plane away from this axis, one could be led to believe that only the perturbative series would be necessary. But as it is known from resurgent theory, and evidence was seen, for example, in Ref. [46], the existence of Stokes lines with a positive real component will introduce nonperturbative sectors which need to be added to the original asymptotic perturbative series in the form of a transseries in order to obtain a consistent result.

It would clearly be interesting to apply the ideas of resurgence theory to the case of $\mathcal{N}=4$ SYM. Applications of resurgence for supersymmetric gauge theories were already

\footnotetext{
${ }^{2}$ See for example $\left[\begin{array}{l|l|l|l|l|}26 & 31\end{array}\right]$ for reviews on resurgent analysis and transseries, and 32 for introductions to resurgence in physical settings. For recent applications to topological strings, supersymmetric quantum mechanics and QFTs, see also 3643 .
} 
seen in the case of localisable observables [47] and relations to quantum mechanical systems [48]. Here we turn to a hydrodynamic model which shares some of the simplicity of MIS theory, but contains a richer spectrum of nonhydrodynamic modes in a way which resembles some aspects of what is known about $\mathcal{N}=4 \mathrm{SYM}$.

It is important to recall here the philosophy behind Ref. [5]: models like MIS are regarded as means of generating the hydrodynamic gradient expansion which is then analysed as if it came from a microscopic theory. Specifically, we will study a hydrodynamic theory which contains analogs of quasinormal modes whose frequencies possess both real and imaginary parts, as is the case for $\mathcal{N}=4 \mathrm{SYM}$ (and unlike MIS). In such a case one would expect that the singularities of the Borel transform would be off the real axis. The simplest such example is one of the models put forth in [49], where nonhydrodynamic modes corresponding to quasinormal modes of $\mathcal{N}=4 \mathrm{SYM}$ were incorporated into a MIS-like theory. This model generates the same hydrodynamic expansion as MIS theory up to second order in gradients (higher orders differ, of course). We show that also in this case one can identify attractor behaviour which sets in well before the hydrodynamic limit of large times.

We study the hydrodynamic series in this model in the spirit of Ref. [5] and find a similar picture, albeit with novel elements. The hydrodynamic series is divergent and its summation requires exponentially suppressed corrections reflecting in a quantitative manner the spectrum of nonhydrodynamic modes present. These exponential corrections to the hydrodynamic series can be viewed as a completion to a transseries. By using the formalism elaborated in [33] we show that the divergent series satisfy relations expected on the basis of resurgence theory. From this perspective there is a novel aspect: the "actions" are complex, as is the leading nonanaliticity exponent. This introduces some technical difficulties in applying convergence acceleration. The physical reason for these features is, however, entirely clear: they correspond to the fact that the nonhydrodynamic modes present in this theory are not purely decaying (i.e., the quasinormal mode frequencies are not purely imaginary). 
The structure of this paper is as follows. We start by reviewing the important aspects of hydrodynamic theories in Section 2, followed by more specific properties of the MIS causal hydrodynamic theory in Section 3 . Section 4 then presents the natural contact between resurgence and the ambiguity cancellation of the MIS theory (reviewing the results of Ref. [5] in light of Refs. [33, 23] ) as a warmup example toward the extended theories of hydrodynamics. Section 5 introduces the hydrodynamic model which we will consider in the main part of this article. The application of resurgence techniques to this theory is the main focus of our work and is described in Section 6. We will close with a brief summary and ideas for the future in Section 7 .

\section{Hydrodynamics}

Phenomenological equations of hydrodynamics are designed to reproduce the gradient expansion of the energy-momentum tensor in some microscopic theory up to some order (typically 1 or 2 ). The evolution equations are the conservation equations

$$
\nabla_{\mu} T^{\mu \nu}=0
$$

of the energy-momentum tensor expressed in terms of the hydrodynamics variables. Specifically, the energy-momentum tensor in the hydrodynamic theories considered here can be presented as

$$
T^{\mu \nu}=\mathcal{E} u^{\mu} u^{\nu}+\mathcal{P}(\mathcal{E})\left(\eta^{\mu \nu}+u^{\mu} u^{\nu}\right)+\Pi^{\mu \nu},
$$

where $\Pi^{\mu \nu}$ is the shear stress tensor (discussed in detail below), $\mathcal{E}$ is the energy density and $\mathcal{P}$ is the pressure, expressed in terms of the energy density by an assumed equation of state. In conformal theories in $d=4$ dimensions it takes the form

$$
\mathcal{P}(\mathcal{E})=\frac{1}{3} \mathcal{E}
$$

The energy density $\mathcal{E}$ is often expressed in terms of the "effective temperature" $T \sim \mathcal{E}^{1 / 4}$. The field $u$ is the flow velocity, defined as a timelike eigenvector of the energy-momentum 
tensor. The spacetime dependent energy density (or effective temperature) and flow velocity are the hydrodynamic variables, the evolution of which one wishes to describe. Their precise definition away from equilibrium is what constitutes a choice of hydrodynamic frame (see, e.g., Ref. [50]). We adopt the Landau frame, which means that we impose the condition that the shear stress tensor is transverse to the flow:

$$
u_{\mu} \Pi^{\mu \nu}=0
$$

The hydrodynamic gradient expansion is the approximation of $\Pi^{\mu \nu}$ by a series of terms, graded by the number of spacetime gradients of the hydrodynamic fields $u^{\mu}$ and $T$.

To proceed, it is highly advantageous to exploit to the fullest the constraints imposed by conformal symmetry. This desire has led to the development of the so-called Weylcovariant formulation [51] of conformal relativistic hydrodynamics, in which the evolution equations assume a very compact form. We will not review this formalism here, but we will mention some of its basic features. The essential idea is to introduce a (nondynamical) "Weyl connection"

$$
\mathcal{A}_{\mu}=u^{\lambda} \nabla_{\lambda} u_{\mu}-\frac{1}{3} \nabla_{\lambda} u^{\lambda} u_{\mu}
$$

to define a derivative operator, denoted here by $\mathcal{D}_{\mu}$, which is covariant under Weyl transformations (spacetime dependent rescalings) of the metric. The action of the Weylcovariant derivative depends on the tensor on which it acts. A general formula can be found in Ref. [51].

It will also be convenient to define

$$
\mathcal{D} \equiv u^{\mu} \mathcal{D}_{\mu}
$$

and

$$
\sigma^{\mu \nu}=\mathcal{D}^{\mu} u^{\nu}+\mathcal{D}^{\nu} u^{\mu}, \quad \omega^{\mu \nu}=\mathcal{D}^{\mu} u^{\nu}-\mathcal{D}^{\nu} u^{\mu}
$$

These objects are transverse and transform homogeneously under Weyl transformations [51].

The Landau-Lifschitz formulation of relativistic viscous hydrodynamics [52] asserts that

$$
\Pi^{\mu \nu}=-\eta \sigma^{\mu \nu}
$$


where $\eta$ is the shear viscosity. Unfortunately, the resulting theory does not have a well-posed initial value problem due to superluminal signal propagation 53,54. The same problem will occur if on the right-hand side of Eq. (8) one includes any finite number of terms graded by the number of derivatives of $T$ and $u^{\mu}$. In principle these problems appear at short distances, where hydrodynamics is not expected to apply [55, 56], but for practical applications this is no consolation because acausality leads to numerical instabilities. For practical purposes it is therefore necessary to replace Eq. (8) by a prescription which effectively generates all orders in the gradient expansion.

\section{$3 \quad$ MIS causal hydrodynamics}

MIS theory resolves the causality problem by promoting the shear stress tensor $\Pi^{\mu \nu}$ to an independent dynamical field which satisfies a relaxation type differential equation [8,9] chosen to augment the conservation law Eq. (1). Consistency with the gradient expansion requires that terms of at least second order be included, since the derivative of the shear stress tensor is of second order.

If all terms admitted by symmetry are incorporated, the leading terms in the gradient expansion of the shear stress tensor can be written as

$$
\Pi^{\mu \nu}=-\eta \sigma^{\mu \nu}+\eta \tau_{\Pi} \mathcal{D} \sigma^{\mu \nu}+\lambda_{1} \sigma_{\lambda}^{<\mu} \sigma^{\nu>\lambda}+\lambda_{2} \sigma_{\lambda}^{<\mu} \omega^{\nu>\lambda}+\lambda_{3} \omega^{<\mu}{ }_{\lambda} \omega^{\nu>\lambda},
$$

where $\left\langle\ldots>\right.$ denotes symmetrization and subtracting the trace, and $\tau_{\Pi}$ and $\lambda_{i}$ are phenomenological parameters [1] (the second-order transport coefficients). If the energymomentum tensor is calculated in some microscopic conformal theory and expressed in terms of the hydrodynamic variables up to second order in gradients, the result will be of the form of Eq. (9) with some specific values of the transport coefficients. It has, for example, been obtained as the long-wavelength effective description of strongly coupled $\mathcal{N}=4$ SYM plasma in the framework of the AdS/CFT correspondence [7, 57, 1, 58.

The main idea of treating hydrodynamics as an effective theory is to write down an evolution equation, the gradient expansion of which generates Eq. (9), together with 
additional terms which are of third order and above. This can be done by eliminating $\sigma^{\nu \lambda}$ in the second-order terms in favor of $\Pi^{\mu \nu}$ using Eq. (8). The result can be written as

$$
\left(\tau_{\Pi} \mathcal{D}+1\right) \Pi^{\mu \nu}=-\eta \sigma^{\mu \nu}+\frac{\lambda_{1}}{\eta^{2}} \Pi_{\lambda}^{<\mu} \Pi^{\nu>\lambda}-\frac{\lambda_{2}}{\eta} \Pi_{\lambda}^{<\mu} \omega^{\nu>\lambda}+\lambda_{3} \omega^{<\mu}{ }_{\lambda} \omega^{\nu>\lambda} .
$$

Solving this iteratively yields Eq. (9) up to higher-order terms, as desired. The coefficients of these higher-order terms will all be expressed in terms of the second-order transport coefficients which appear explicitly in Eq. 10.

Linearization of the resulting theory reveals a single, purely decaying, nonhydrodynamic mode in addition to hydrodynamic modes [1]. This mode (which we refer to as the MIS mode) decays on a scale set by $\tau_{\Pi}$. Furthermore, the resulting theory is causal as long as $T \tau_{\Pi} \geq \eta / s$. This approach has enjoyed great success in describing the evolution of quark-gluon plasma [59].

In Ref. [5], the special case of Bjorken flow [6] was considered, and we do the same in our work. Due to a very high degree of symmetry imposed, the hydrodynamic equations reduce to a set of ordinary differential equations. The symmetry in question, boost invariance, can be taken to mean that in proper time-rapidity coordinates $\tau, y$ (related to Minkowski coordinates $t, z$ by the relations $t=\tau \cosh y$ and $z=\tau \sinh y$ ) the energy density, flow velocity and shear stress tensor depend only on the proper time $\tau$. The MIS equations Eq. (10) then reduce to

$$
\begin{aligned}
\tau \dot{\epsilon} & =-\frac{4}{3} \epsilon+\phi \\
\tau_{\Pi} \dot{\phi} & =\frac{4 \eta}{3 \tau}-\frac{\lambda_{1} \phi^{2}}{2 \eta^{2}}-\frac{4 \tau_{\Pi} \phi}{3 \tau}-\phi
\end{aligned}
$$

where the dot denotes a proper time derivative and $\phi \equiv-\Pi_{y}^{y}$, the single independent component of the shear stress tensor.

In a conformal theory $\epsilon \sim T^{4}$ and the transport coefficients satisfy

$$
\tau_{\Pi}=\frac{C_{\tau \Pi}}{T}, \quad \lambda_{1}=C_{\lambda_{1}} \frac{\eta}{T}, \quad \eta=C_{\eta} s
$$

where $s$ is the entropy density and $C_{\tau \Pi}, C_{\lambda_{1}}, C_{\eta}$ are dimensionless constants. In the case of 
$\mathcal{N}=4$ SYM their values are known from fluid-gravity duality [58]:

$$
C_{\tau \Pi}=\frac{2-\log (2)}{2 \pi}, \quad C_{\lambda_{1}}=\frac{1}{2 \pi}, \quad C_{\eta}=\frac{1}{4 \pi} .
$$

To simplify the discussion we will consider the case $C_{\lambda_{1}}=0$. This choice does not modify the nonydrodynamic sector in a qualitative way, so our study of resurgence is not affected. The hydrodynamic theory still matches $\mathcal{N}=4 \mathrm{SYM}$ at the level of first-order (viscous) hydro, which is physically by far the most significant point.

Using Eq. (12) one can turn the system of equations Eq. (11) into a single second-order differential equation for the proper time dependence of the temperature $T(\tau)$. It proves fruitful to introduce the dimensionless variables

$$
w=T \tau, \quad f=\frac{\tau}{w} \frac{d w}{d \tau} .
$$

In terms of these, the second-order ordinary differential equation for $T(\tau)$ implies a first-order equation for $f(w)$,

$$
C_{\tau \Pi} f f^{\prime}+4 C_{\tau \Pi} f^{2}+\left(w-\frac{16 C_{\tau \Pi}}{3}\right) f-\frac{4 C_{\eta}}{9}+\frac{16 C_{\tau \Pi}}{9}-\frac{2 w}{3}=0,
$$

where $f^{\prime}$ stands for the derivative of $f(w)$ with respect to $w$. It is this equation which is the starting point for our analysis of MIS theory.

The late proper time behaviour of the system is governed by hydrodynamics. In terms of the dimensionless variable $w$ this translates to the limit $w \rightarrow \infty$. One can easily determine the coefficients of the series solution valid for large $w$ :

$$
f(w)=\frac{2}{3}+\frac{4 C_{\eta}}{9 w}+\frac{8 C_{\eta} C_{\tau \Pi}}{27 w^{2}}+O\left(\frac{1}{w^{3}}\right) .
$$

This expansion corresponds to the hydrodynamic gradient expansion [60]. By examining the behaviour of the coefficients in Eq. (16) one can show that the series as divergent. This fact reflects the presence of the nonhydrodynamic MIS mode. As shown in [5] the series solution can be summed using Borel techniques by incorporating exponential corrections to the hydrodynamic expansion. The result is a transseries, as described in detail in the following section. 


\section{Resurgence and ambiguity cancellation}

As an introduction to the methods of resurgence theory we first consider the case of MIS reviewed in the previous section. Unlike the analysis presented in Ref. [5], we will not discuss ambiguity cancellation at the level of the analytic continuation of the Borel transform. We will instead make use of the consistency conditions derived from alien calculus (for a recent review and derivations of the formulae used in this Section see Refs. [33, 30, 35]). As was already seen in Ref. [5], this example presents some remarkable resurgent properties, while being a very simple application of the expressions derived in Ref. [33, 23].

In order to capture the full solution to the first-order differential equation Eq. (15) from a perturbative expansion, one needs a transseries Ansatz with one parameter

$$
f(w, \sigma)=\sum_{n=0}^{+\infty} \sigma^{n} \mathrm{e}^{-n A w} \Phi_{n}(w)
$$

where $\sigma$ is a parameter to be fixed by the physical properties of our system - in our case these are reality and initial conditions. The constant $A$ is often referred to as the instanton action, due to its interpretation in applications to perturbation expansions in quantum field theory 12 18.

The $\Phi_{n}(w)$ are perturbative expansions around the nonperturbative, exponentially suppressed sectors with contributions weighted by $\mathrm{e}^{-n A w}$

$$
\Phi_{n}(w)=w^{\beta_{n}} \sum_{k=0}^{+\infty} a_{k}^{(n)} w^{-k} .
$$

The "instanton" action $A$ and coefficients $\beta_{n}$ (the first associated with the position of the cuts appearing in the Borel plane, and the latter associated with the type of these branch cuts) were determined in Ref. [5] to be

$$
A=\frac{3}{2} C_{\tau \Pi}, \quad \beta_{n} \equiv n \beta=-n \frac{C_{\eta}}{C_{\tau \Pi}} .
$$

These coefficients, as well as the perturbative coefficients $a_{k}^{(n)}$ can be determined iteratively by substituting the transseries Ansatz (18) into the differential equation (15). For the 
perturbative series $\Phi_{0}(w)$ this leads to

$$
\begin{aligned}
a_{0}^{(0)} & =\frac{2}{3} \\
a_{1}^{(0)} & =\frac{4 C_{\eta}}{9} \\
a_{k+1}^{(0)} & =C_{\tau \Pi}\left(\frac{16}{3} a_{k}^{(0)}-\sum_{n=0}^{k}(4-n) a_{k-n}^{(0)} a_{n}^{(0)}\right), \quad k>1 .
\end{aligned}
$$

This recursion relation makes it manifest that the series is indeed divergent.

The expansions $\Phi_{n}(w)$ are asymptotic, and the coefficients $a_{k}^{(n)}$ were seen to grow factorially for large enough order $k$. The corresponding Borel transforms, schematically of the form ${ }^{3}$

$$
\mathcal{B}\left[\Phi_{n}\right](s)=\sum_{m=m_{\min }}^{+\infty} a_{m}^{(n)} \frac{s^{m-n \beta-1}}{\Gamma(m-n \beta)},
$$

have a nonzero radius of convergence. The radius of convergence is in fact given by the position of the first branch cut in the Borel plane, which is at a distance $s=A$. Following the analysis presented in Ref. [33] (see Section 2 of this paper for more details), we know that in the case of a one-parameter transseries with real positive instanton action $A$, the sectors $\Phi_{0}, \Phi_{1}$ will have cuts starting at positions $s=\ell A$ for $\ell \geq 1$ in the positive real axis, while the sectors $\Phi_{n}$ with $n \geq 2$ will have cuts both in the negative and positive real lines on the Borel plane: a finite number in the negative real axis at $s=\ell_{1} A$ with $\ell_{1}=1, \cdots, n-1$, and an infinite number in the positive real axis at $s=\ell_{2} A$ with $\ell_{2} \geq 1$

Given the Borel transforms, Eq. (21), one can use suitable Padé approximants (see Ref. [33]) and resum each sector via the Laplace transform. The resummation can be easily performed in directions $\theta$ in the complex plane where the Borel transforms $\mathcal{B}\left[\Phi_{n}\right]$ do not have singular behaviour:

$$
\mathcal{S}_{\theta} \Phi_{n}(w)=\int_{0}^{+\infty \mathrm{e}^{\mathrm{i} \theta}} d s \mathrm{e}^{-s w} \mathcal{B}\left[\Phi_{n}\right](s) .
$$

\footnotetext{
${ }^{3}$ The rule is to substitute $w^{-\alpha} \rightarrow s^{\alpha-1} / \Gamma(\alpha)$, but one needs to remove any initial terms with $\alpha<0$, and add them separately at a later stage: hence the $m_{\min }$ introduced in Eq. 21). This does not change the asymptotic nature of the series.
} 
This resummation can then be trivially analytically continued up to the singular directions in the Borel plane, also known as Stokes lines. The resummed transseries can then be defined by

$$
\mathcal{S}_{\theta} f(w, \sigma)=\sum_{n=0}^{+\infty} \sigma^{n} \mathrm{e}^{-n A w} \mathcal{S}_{\theta} \Phi_{n}(w) .
$$

The transseries parameter $\sigma$ is free at this stage. Its (in general complex) value can be determined by enforcing some physical constraints on the transseries - in this case suitable initial and reality conditions for Eq. 40.

For physical reasons, we are ultimately interested in real and positive values of the expansion parameter $w$. For the particular case in this Section, we have one further problem: the Borel transforms of the perturbative and nonperturbative sectors have branch cuts in the positive real axis starting at positions $s=\ell A$. This means that the positive real axis is a Stokes line, a singular direction in the complex plane where the Stokes phenomenon occurs. The Laplace transform in (22) is ill defined, because of these branch cuts, and we have to define lateral resummations by avoiding these singularities either from above or from below the real axis:

$$
\mathcal{S}_{ \pm} \Phi_{n}(w)=\int_{0}^{+\infty \mathrm{e}^{ \pm \mathrm{i} \epsilon}} d s \mathrm{e}^{-s w} \mathcal{B}\left[\Phi_{n}\right](s) .
$$

This introduces a nonperturbative ambiguity: if the coefficients $a_{m}^{n}$ are real, then the difference between these two lateral resummations for each sector is pure imaginary and of the order of $\mathrm{e}^{-A w}$. For example, starting with the lateral resummation above the real axis, we can define its real and imaginary contribution

$$
\mathcal{S}_{+} \Phi=\frac{1}{2}\left(\mathcal{S}_{+}+\mathcal{S}_{-}\right) \Phi+\frac{1}{2}\left(\mathcal{S}_{+}-\mathcal{S}_{-}\right) \Phi \equiv \mathcal{S}_{R} \Phi+\mathrm{i} \mathcal{S}_{I} \Phi
$$

where $\mathcal{S}_{I} \Phi_{n}(w) \sim \mathrm{e}^{-A w}$. One is interested in having a nonambiguous real transseries solution, i.e., a transseries of the type (23) but where now the resummation should be thought of as one of the lateral resummations $\mathcal{S} \rightarrow \mathcal{S}_{ \pm}$. Because $\sigma$ is a complex number, the real and imaginary contributions from each sector $\mathcal{S}_{R, I} \Phi_{n}$ will mix with the real and imaginary part of the parameter $\sigma \equiv \sigma_{R}+\mathrm{i} \sigma_{I}$, and one can determine the total real and 
imaginary contributions to the transseries coming from every sector. This was done in detail in [23].

Due to the resurgent properties of the transseries, choosing the parameter $\sigma$ properly will cancel the imaginary "ambiguous" contribution to the transseries, leaving us with a nonambiguous real result. This procedure coincides with the so-called median resummation. In 23 it was seen that for a one-parameter transseries with real coefficients and singularities in the Borel plane lying in the positive real axis $4^{4}$ the median resummation was achieved by setting the imaginary part of the transseries parameter to

$$
\mathrm{i} \sigma_{I}=-\frac{1}{2} S_{1}
$$

Here $S_{1}$ is the so-called Stokes constant associated with the Stokes transition across the positive real axis. The real part of the parameter $\sigma$ does not get fixed by these requirements, and remains as an integration constant, to be fixed by some initial condition. The nonambiguous real transseries solution to this problem is therefore given by

$$
\mathcal{S}_{R} f=\mathcal{S}_{+} f\left(w, \sigma_{R}-\frac{1}{2} S_{1}\right)=\sum_{n=0}^{+\infty}\left(\sigma_{R}-\frac{1}{2} S_{1}\right)^{n} \mathrm{e}^{-n A w} \mathcal{S}_{+} \Phi_{n}(w) .
$$

The Stokes constant can be determined directly by using resurgence formulae predictions for the large-order behaviour of the perturbative series (as well as higher sectors). From the resurgent analysis of the one-parameter transseries, it was seen in Ref. [33 that the discontinuity of the sectors $\Phi_{k}$ in the positive real direction $w=|w| \mathrm{e}^{\mathrm{i} \theta}$ with $\theta=0$ is given by

$$
\operatorname{Disc}_{0} \Phi_{k}=-\sum_{\ell=1}^{+\infty} \frac{(k+\ell) !}{k ! \ell !}\left(S_{1}\right)^{\ell} \mathrm{e}^{-\ell A w} \Phi_{k+\ell}(w) .
$$

For the particular case of the perturbative expansion we need only to set $k=0$. For $k \geq 2$ the sectors $\Phi_{k}$ will also have discontinuities in the direction $\theta=\pi$. The full expressions for these discontinuities were also derived in Ref. [33] (in Section 2), but given the length of

\footnotetext{
${ }^{4}$ Recall that higher nonperturbative sectors $\Phi_{n}$ with $n \geq 2$ will naturally have singularities also in the negative real line, as it is expected from a one-parameter transseries. This is a natural part of the resurgent analysis and its consequences are already integrated in the analysis presented in Refs. 33,23 .
} 
such expressions we refer the reader to that reference for more details. The fact that our transseries is resurgent translates directly to the existence of large-order relations between the coefficients $a_{k}^{(n)}$ and $a_{k^{\prime}}^{(m)}$ of neighbouring sectors $n, m$. These large-order relations can be derived using Cauchy's theorem,

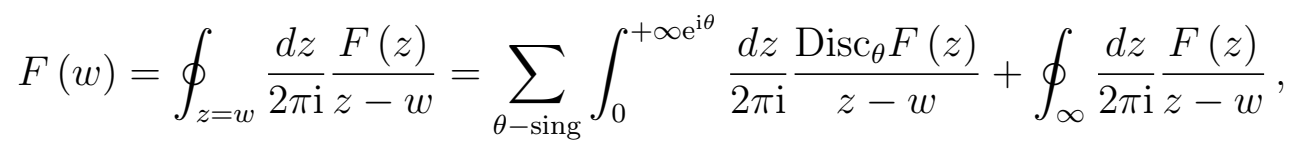

where the sum is over all singular directions $\theta$ of the asymptotic expansion $F(w)$. On the rhs, we have deformed the contour of integration to encircle all the discontinuities (associated with the singular directions $\theta$ ) and the contribution at infinity. Under certain conditions [11, 61], the contribution at infinity can be seen to vanish by scaling arguments and we are left with the integration over the discontinuities of $F(w)$.

As an example choose the perturbative sector $\Phi_{0}$ and use variables $x=w^{-1} \ll 1$. We can easily write

$$
x \Phi_{0}(x)=\oint_{z=x} \frac{d z}{2 \pi \mathrm{i}} \frac{z \Phi_{0}(z)}{z-x}=\int_{0}^{+\infty} \frac{d z}{2 \pi \mathrm{i}} \frac{z \operatorname{Disc}_{0} \Phi_{0}(z)}{z-x} .
$$

In the above formula we used that the perturbative series has only a discontinuity in the direction $\theta=05^{5}$ We can now use (28) and expand both sides for small $x$. Comparing equal powers of $x$ we find

$$
a_{m}^{(0)} \simeq-\sum_{k=1}^{+\infty} \frac{\left(S_{1}\right)^{k}}{2 \pi \mathrm{i}} \frac{\Gamma(m+k \beta)}{(k A)^{m+k \beta}} \sum_{h=0}^{+\infty} a_{h}^{(k)} \prod_{\ell=1}^{h} \frac{k A}{(m+k \beta-\ell)}, m \gg 1 .
$$

This is a large-order relation with connects coefficients of the perturbative series $a_{m}^{(0)}$ for large-order $m$ with coefficients of the one-instanton series $a_{h}^{(1)}$ at low order, up to contributions of the two-instanton series $a_{h}^{(2)}$ exponentially suppressed by $2^{-m}$, and so on. Note that each of the sums appearing above is asymptotic. Similar expressions have been derived for the coefficients of the other sectors, and can be found in Ref. [33]. For the coefficients of the higher sectors $\Phi_{k}, k \geq 2$ other Stokes constants will appear in the

\footnotetext{
${ }^{5}$ Recall again that for $F(w)=\Phi_{k}(w)$ with $k=0,1$ we have only one singular direction $\theta=0$, while for $k \geq 2$ we have two singular directions $\theta=0, \pi$.
} 
large-order relations, because of the discontinuity in the direction $\theta=\pi$ (see Section 2 of Ref. [33] for more details).

Returning to the perturbative series, we can write the large-order relations more explicitly in the following way:

$$
\begin{aligned}
\frac{2 \pi \mathrm{i} A^{m+\beta}}{\Gamma(m+\beta)} a_{m}^{(0)} & \simeq-S_{1} \sum_{h=0}^{+\infty} a_{h}^{(1)} \prod_{\ell=1}^{h} \frac{A}{(m+\beta-\ell)}+\mathcal{O}\left(2^{-m}\right) \\
& \simeq-S_{1}\left(a_{0}^{(1)}+\frac{A}{m} a_{1}^{(1)}+\frac{A^{2} a_{2}^{(1)}-A(\beta-1) a_{1}^{(1)}}{m^{2}}+\cdots\right)+\mathcal{O}\left(2^{-m}\right) .
\end{aligned}
$$

To obtain the second line we expanded the first line for large $m$. Now it becomes clear how one can determine the Stokes constant. Having calculated the coefficients $a_{m}^{(0)}$ and $a_{n}^{(1)}$ iteratively from the differential equation, we can now analyse the convergence of the lhs to the Stokes constant (times the value of $a_{0}^{(1)}$ ), and thus determine $S_{1}$.

To carry out this calculation we make use of the accelerated convergence of the Richardson transforms [62 64 (see Fig. 1). This leads to the determination of the Stokes constant as $S_{1}=-0.00547029853$ i, which matches the result in Ref. [5].

It is important to note that we can check the predictions obtained by resurgence techniques for the large-order behaviour of the perturbative series even without the knowledge of the Stokes constant. To do so, we analyse the convergence of the ratio of coefficients to the predicted values

$$
R_{m} \equiv \frac{a_{m+1}^{(0)} A}{a_{m}^{(0)} m}
$$

On the basis of the analysis we have presented above, we expect

$R_{m} \simeq\left(1+\frac{\beta}{m}\right)\left(1-\frac{A}{m} \frac{a_{1}^{(1)}}{a_{0}^{(1)}}+\frac{A^{2}\left(a_{1}^{(1)} / a_{0}^{(1)}\right)^{2}+(2 \beta-1) A a_{1}^{(1)} / a_{0}^{(1)}-2 A^{2} a_{2}^{(1)} / a_{0}^{(1)}}{m^{3}}+\cdots\right)$.

This quantity is clearly of the form

$$
R_{m} \simeq \sum_{k=0}^{+\infty} \frac{c_{k}}{m^{k}}
$$




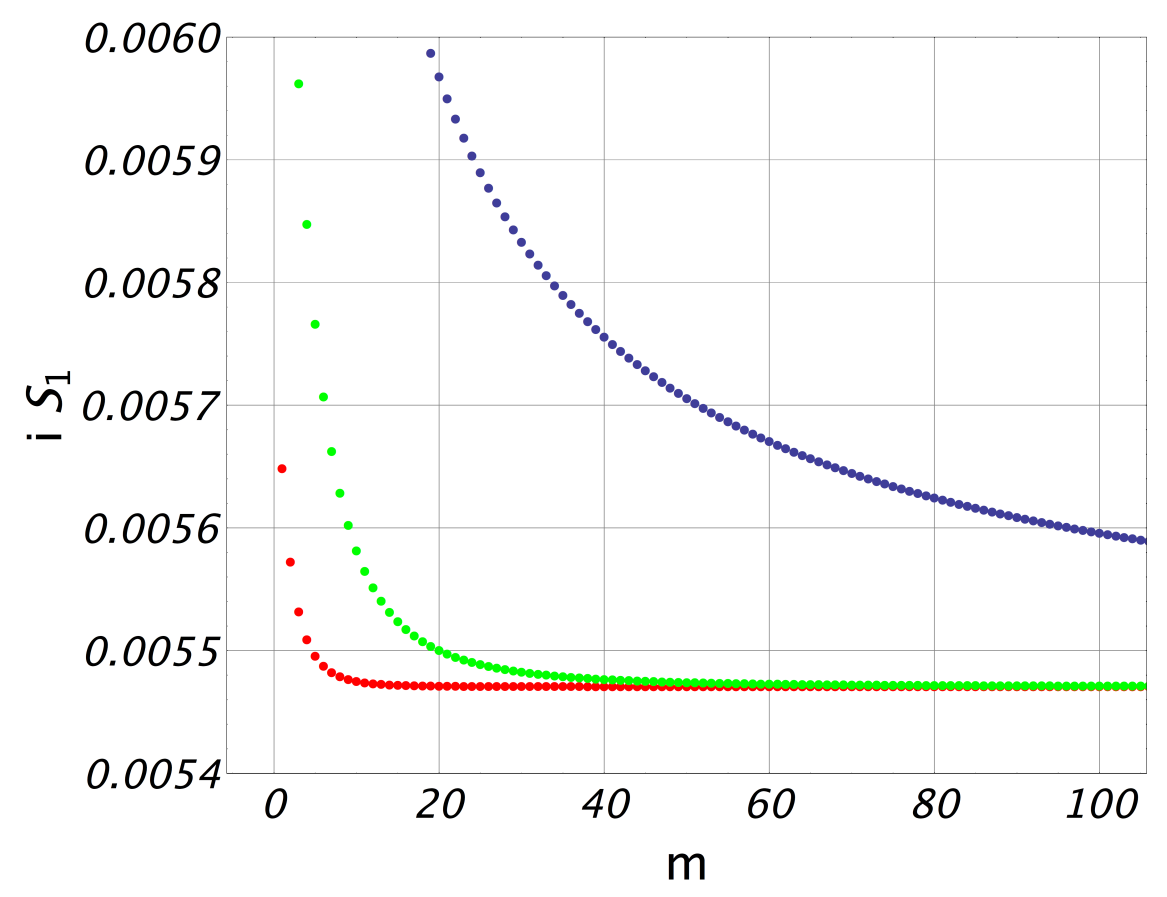

Figure 1: Convergence of the large-order perturbative series (in blue) to the Stokes constant, using Richardson transforms of order 2 (green) and 5 (red).

where the coefficients $c_{k}$ are directly determined from the large $m$ expansion of the large-order relation given above. This makes it possible to use Richardson transforms to accelerate convergence. As seen in Fig. 2, the ratio Eq. (33) converges to unity $c_{0}=1$ rather quickly. If the Richardson transform (of order 10) is used, already at $m=20$ this ratio differs from unity no more than one part in $10^{8}$ (and at $m=100$ the Richardson transform of order 10 has an error of $\left.10^{-16}\right)$.

Finally, note that one can easily check for consistency the value of any coefficient $c_{k}$ predicted by resurgence by checking the convergence of

$$
\widetilde{R}_{m}(k) \equiv\left(R_{m}-\sum_{r=0}^{k-1} \frac{c_{r}}{m^{r}}\right) m^{k} \simeq c_{k}+\mathcal{O}\left(m^{-1}\right) .
$$

In Fig. 3 this convergence can be seen to the predicted value of $c_{5}=-31.1456818997329$. For a Richardson transform of order 5 , the error of the predicted value is $10^{-8}$.

This Section has served as a warmup for some of the resurgence techniques that we will be using in what follows. We needed to resolve a nonperturbative ambiguity due to 


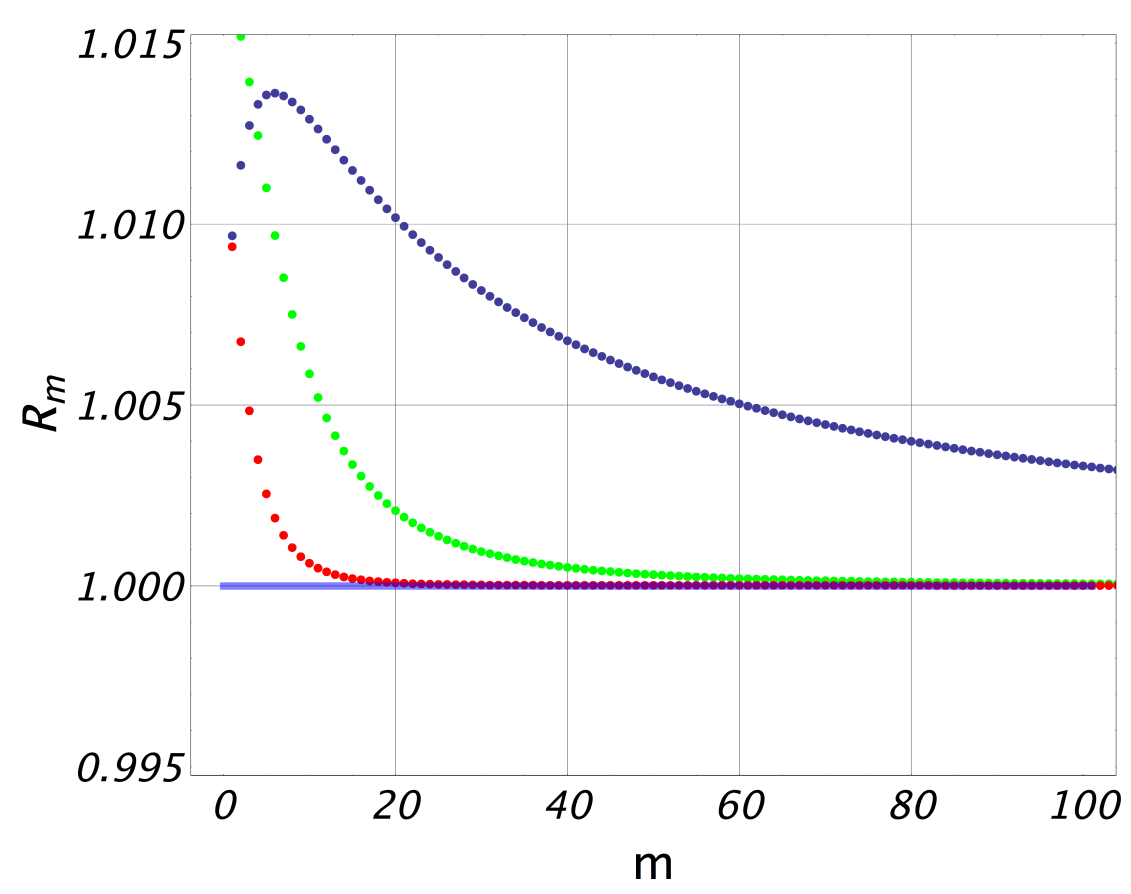

Figure 2: Convergence of the ratio 32 , in blue, to the leading term in the large-order relation $c_{0}=1$ (light blue). The accelerated convergence is shown using Richardson transforms of order 2 (green) and 5 (red).

a singularity on the positive real axis and obtained results consistent with Ref. [5]. The following Section applies resurgence techniques to an extended hydrodynamic theory which involves a second-order differential equation with two Stokes lines in the complex plane, both away from the positive real axis. Even though there will be no ambiguities in the resummation procedure along the positive real axis, resurgence plays a determinant role in the construction of the full transseries answer. The methods of resurgence that will be used to study this problem are a generalisation of what was presented in this Section and in Ref. [33] (a review of nonlinear resurgent transseries with two and more parameters can be found in Ref. 35]).

Note finally that one can of course solve Eq. (15) numerically. These exact solutions rapidly converge to an attractor which determines universal behaviour which emerges after nonhydrodynamic degrees of freedom decay. The transseries solution captures this behaviour: adding just the lowest order in the transseries makes it possible to match the 


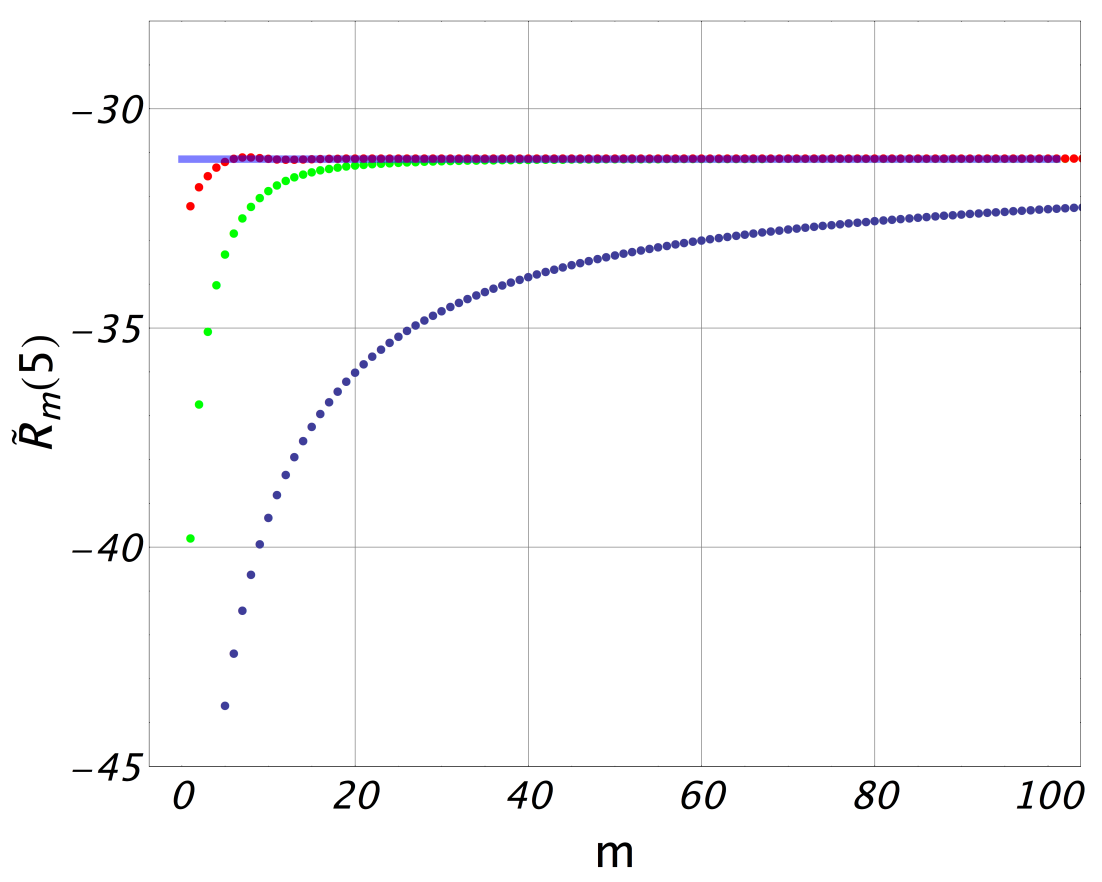

Figure 3: Convergence of the ratio 32 , in blue, to the large-order relation coefficient $c_{5}$ (light blue). The accelerated convergence is shown using Richardson transforms of order 2 (green) and 5 (red).

attractor solution by choosing the real part of the transseries parameter $\sigma$ appropriately [5].

While this work was in preparation, the authors became aware of Ref. 65] which has some overlap with this Section.

\section{$5 \quad$ Extended theories of hydrodynamics}

As reviewed in Section 3, MIS theory contains a single purely damped nonhydrodynamic mode, the presence of which is reflected in the divergence of the gradient expansion. The occurrence of this mode is enough to furnish a causal hydrodynamic theory close to local equilibrium. This theory has been very successful in describing the evolution of quark-gluon plasma produced in heavy ion collisions, beginning with proper times of less than a fermi/c. It has, however, been noted by many authors $66,60,67$ that the pressure anisotropy at these early times is still very large, and the system is not close to equilibrium. It is 
natural to suspect that the nonhydrodynamic MIS mode not only regulates the causality and stability issues of Navier-Stokes hydrodynamics, but contributes in a very nontrivial way to the physical implications of this model. This provides strong motivation to try to understand better the role of nonhydrodynamic modes, and how they can be matched with a microscopic description. For modeling early nonequilibrium dynamics one would expect that incorporating further nonhydrodynamic degrees of freedom should provide a better description.

A significant step leading in this direction was taken in Ref. [49], where extended hydrodynamic theories were formulated in the context of $\mathcal{N}=4 \mathrm{SYM}$. These theories attempted to match the effective theory to the pattern of the least damped black brane quasinormal modes which govern the approach to hydrodynamics.

In this paper we focus on the simplest model discussed in Ref. [49] in which there is a pair nonhydrodynamic modes which are not purely decaying. The familiar relaxation equation MIS theory, Eq. (10), is replaced by

$$
\left(\left(\frac{1}{T} \mathcal{D}\right)^{2}+2 \Omega_{I} \frac{1}{T} \mathcal{D}+|\Omega|^{2}\right) \Pi^{\mu \nu}=-\eta|\Omega|^{2} \sigma^{\mu \nu}-c_{\sigma} \frac{1}{T} \mathcal{D}\left(\eta \sigma^{\mu \nu}\right)+\ldots,
$$

where the ellipsis denotes contributions of second and higher order in gradients. The parameter

$$
\Omega \equiv \Omega_{R}+i \Omega_{I}
$$

is the complex "quasinormal mode" frequency. The coefficient $c_{\sigma}$ affects the region of stability in parameter space [49]. By solving Eq. (37) in the gradient expansion one can also check that $c_{\sigma}$ contributes to second order transport coefficients. However, in our work this coefficient does not play a qualitative role and we will set it to zero.

The appearance of the second derivative in Eq. (37) is what leads to nonhydrodynamic modes which are not purely decaying. Indeed, the linearization of equations Eq. (1) and Eq. (37) around flat space reveals a pair of nonhydrodynamic modes with complex frequencies $\Omega$ and $-\bar{\Omega}$. In the case of $\mathcal{N}=4$ SYM the leading quasinormal mode frequencies 
have the values 68

$$
\Omega_{R} \approx 9.800, \quad \Omega_{I} \approx 8.629 .
$$

and these are the values we assume in our calculations 6 ,

As in the case of MIS theory, upon imposing boost invariance the hydrodynamic equations reduce to an ordinary differential equation for the temperature. Of course, in the present case, the equation is of third order. Introducing new variables as in Eq. (14) one can rewrite it as a second-order differential equation for the function $f(w)$ :

$$
w f^{2} f^{\prime \prime}+\alpha f f^{\prime}+12 f^{2} f^{\prime}+w f f^{\prime 2}+\frac{\beta+\gamma f+\delta f^{2}+12 f^{3}}{w}=0
$$

where $f^{\prime}$ and $f^{\prime \prime}$ are the first and second derivatives of $f(w)$ with respect to $w$, and

$$
\begin{aligned}
\alpha & \equiv-8+2 w \Omega_{I}, \\
\beta & \equiv-\frac{128}{27}-\frac{32}{27} C \eta C_{\tau \Pi}-\frac{4}{9} w\left(C_{\eta}|\Omega|^{2}-8 \Omega_{I}\right)-\frac{2}{3} w^{2}|\Omega|^{2}, \\
\gamma & \equiv \frac{176}{9}+\frac{4}{3} C_{\eta} C_{\tau \Pi}-\frac{32}{3} w \Omega_{I}+w^{2}|\Omega|^{2}, \\
\delta & \equiv-\frac{80}{3}+8 w \Omega_{I} .
\end{aligned}
$$

This is the analog of Eq. 15 of MIS theory.

For physical reasons it is clear that at late times (large $w$ ) the solution must tend to $2 / 3$, which corresponds to ideal fluid behaviour. It is easy to see analytically that this is indeed the asymptotic solution. One can easily determine the large- $w$ expansion of solutions:

$$
f(w)=\frac{2}{3}+\frac{4 C_{\eta}}{9} \frac{1}{w}+\frac{8 C_{\eta}\left(C_{\tau \Pi}+2 \Omega_{I}\right)}{27|\Omega|^{2}} \frac{1}{w^{2}}+\ldots
$$

As expected, the first two terms coincide with what one obtains in MIS theory (see Eq. (20)), whereas the third term is different. This series can be calculated up to essentially any order and can be shown to be divergent, as discussed in much detail in the following section.

\footnotetext{
${ }^{6}$ The values in Eq. (39) differ from those in Table 1 of Ref. 68. (corresponding to an operator of conformal weight $\Delta=4$ ) by a factor of $2 \pi$.
} 
At early times, which correspond to small values of $w$, one finds a unique real power series solution of the form

$$
f(w)=\frac{8}{9}+\frac{9 C_{\eta}|\Omega|^{2}-8 \Omega_{I}}{3\left(20+9 C_{\eta} C_{\tau \Pi}\right)} w+\ldots
$$

By examining numerical solutions of Eq. 40 it is clear that (similarly to the case of MIS theory) this is the small $w$ behaviour of an attractor solution valid in the entire range of $w$.

Since Eq. (40) is of second order, one must specify both $f$ and $f^{\prime}$ at the initial value of $w$. As seen in Fig. 4, setting initial conditions at various values of $w$ shows that the numerical solutions converge to the attractor. However, unlike in the MIS case, the numerical solutions do not decay monotonically but oscillate around the attractor.

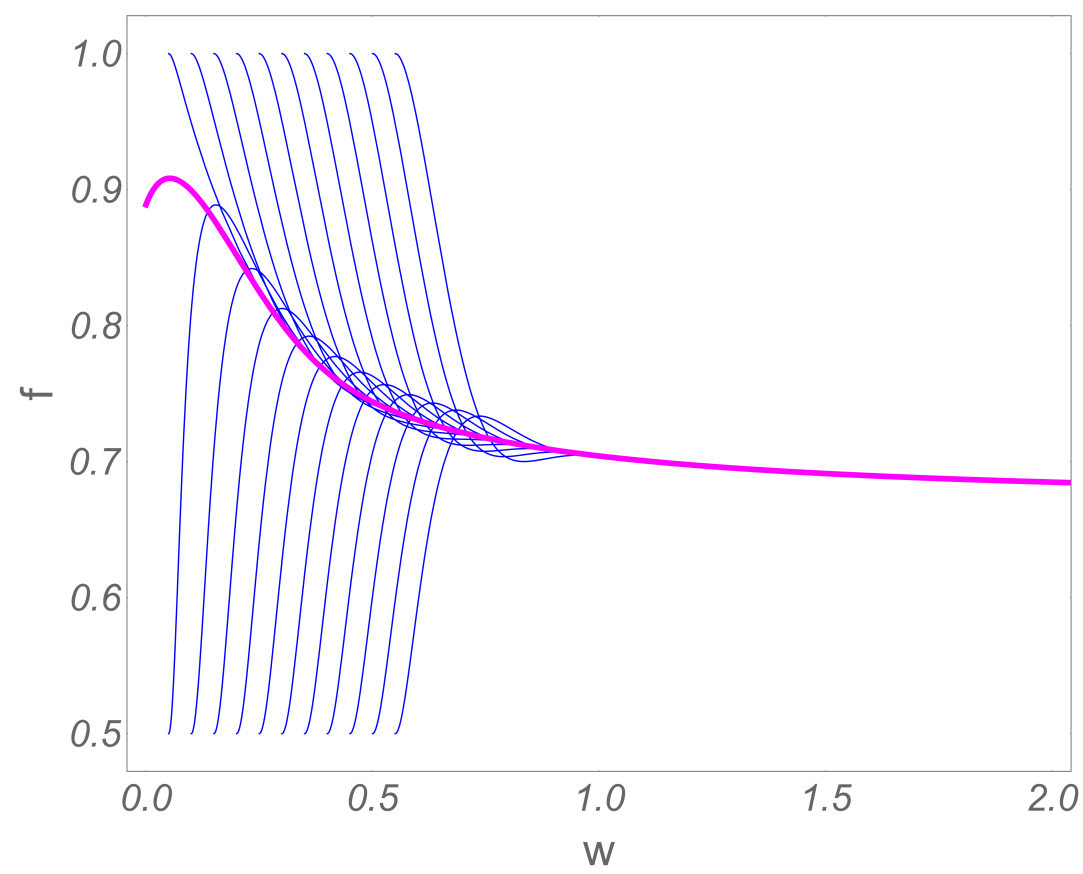

Figure 4: Numerical solutions converge (nonmonotonically) to the numerical attractor (magenta). 


\section{$6 \quad$ Resurgence in extended hydrodynamics}

We are interested in solving Eq. (40) as an expansion in large values of $w \gg 1$. If we use a transseries Ansatz of the type (18) and substitute it into this equation, we easily find two complex conjugate values for the instanton action

$$
A_{ \pm}=\frac{3}{2}\left(\Omega_{I} \pm \mathrm{i} \Omega_{R}\right)
$$

Equivalently, we can write $A_{+}=\frac{3}{2} \mathrm{i} \bar{\Omega}$, while $A_{-}=-\frac{3}{2} \mathrm{i} \Omega$. We then have two types of nonperturbative contributions and thus, following Refs. [33, 35], we find that we need a two-parameter transseries to fully describe the solutions to this equation,

$$
f\left(w, \sigma_{ \pm}\right)=\sum_{n, m=0}^{+\infty} \sigma_{+}^{n} \sigma_{-}^{m} \mathrm{e}^{-\left(n A_{+}+m A_{-}\right) w} \Phi_{(n \mid m)}(w)
$$

where $\Phi_{(n \mid m)}(w)$ are the perturbative expansions in $w^{-1}$ around each sector. The perturbative sector is given by taking $n=m=0$. These expansions are of the form

$$
\Phi_{(n \mid m)}(w)=w^{\beta_{n, m}} \sum_{k=0}^{+\infty} a_{k}^{(n \mid m)} w^{-k}
$$

where the coefficients $\beta_{n, m}$ reflect the type of branch cut singularities in the Borel plane, and the $a_{k}^{(n \mid m)}$ are the expansion coefficients which can be determined iteratively by substituting Eq. (45) into Eq. (40). Assuming furthermore that $\beta_{n, m}=n \beta_{+}+m \beta_{-}$, we find

$$
\beta_{ \pm}=C_{\eta}\left(\Omega_{I} \pm \mathrm{i} \Omega_{R}\right)
$$

together with recursion equations for the coefficients $a_{k}^{(n \mid m)}$. Because $A_{ \pm}$are complex conjugate, as well as $\beta_{ \pm}$, and given that the coefficients of Eq. 40 are all real, we see that that the coefficients $a_{k}^{(n \mid m)}$ will be complex conjugates of $a_{k}^{(m \mid n)}$ (and consequently all $a_{k}^{(n \mid n)}$ will be real).

By studying numerically the behaviour of the coefficients of the perturbative series we see that these grow factorially for large enough order $k$. This is directly related to the behaviour of the Borel transforms. If we define the Borel transform for each sector

$$
\mathcal{B}\left[\Phi_{(n \mid m)}\right](s)=\sum_{k=k_{\min }} a_{k}^{(n \mid m)} \frac{s^{k-\beta_{n, m}-1}}{\Gamma\left(k-\beta_{n, m}\right)},
$$


we find a nonzero radius of convergence and branch cuts starting at positions $s_{ \pm, \ell}=\ell A_{ \pm}$. Note that $k_{\min }$ is the minimum value of $k$ such that every power of $s$ appearing in the Borel transform is non-negative (this does not change the asymptotic properties of the series). In Fig. 5, we see this behaviour for the Borel transform of the perturbative series

$$
\mathcal{B}\left[\Phi_{(0 \mid 0)}\right](s)=\sum_{k=0} a_{k+1}^{(0 \mid 0)} \frac{s^{k}}{\Gamma(k+1)} .
$$

In order to analyse the singularities of the Borel transform, we use the method of Padé approximants, where the series above is approximated by a ratio of polynomials.7 Positions of the zeros of the polynomial in the denominator reflect the singular behaviour of the Borel transform: these poles condense in certain directions, and indicate cuts in the Borel plane.

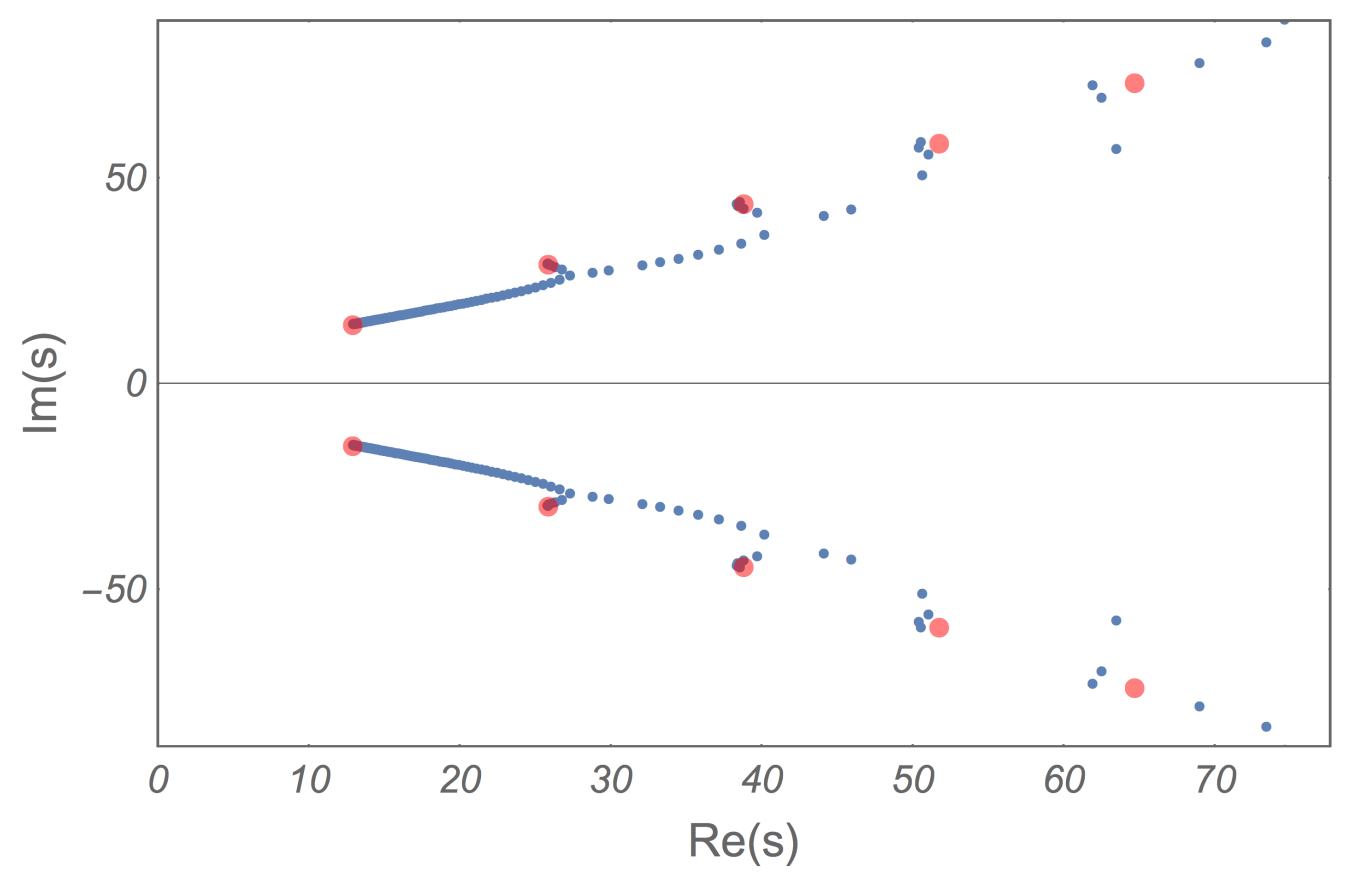

Figure 5: Poles of the diagonal Borel-Padé approximant of order 300 associated with $\Phi_{(0 \mid 0)}$ in the Borel $s$-plane. The red dots indicate values of the multiple instanton actions $\ell A_{ \pm}, \ell \geq 1$.

\footnotetext{
${ }^{7}$ In the diagonal case used here, this ratio is of the same order, half of the number of coefficients determined for the original series (see for example Ref. 33 for more details).
} 
We check the resurgent properties of the transseries Eq. (45) by determining the largeorder behaviour predicted by resurgence for the perturbative series $\Phi_{(0 \mid 0)}$ (the procedure can then be generalised for higher sectors). We first need to determine the associated discontinuities, and then make use of Cauchy's theorem $(29)$, in the same manner as for the one-parameter example previously studied. 8

In the present case we have two singular directions defined by the two actions $A_{ \pm}$:

$$
\theta_{ \pm}= \pm \arctan \left(\frac{\Omega_{R}}{\Omega_{I}}\right) \equiv \pm \theta_{A}
$$

Each of these directions will have a different Stokes constant associated with it, which we will call $S_{ \pm}$. Following the ideas of [33,35] we can write down the discontinuities associated with the singular directions as

$$
\begin{aligned}
& \operatorname{Disc}_{\theta_{+}} \Phi_{(0 \mid 0)}(w)=-\sum_{\ell=1}^{+\infty}\left(S_{+}\right)^{\ell} \mathrm{e}^{-\ell A_{+} w} \Phi_{(\ell \mid 0)}(w), \\
& \operatorname{Disc}_{\theta_{-}} \Phi_{(0 \mid 0)}(w)=-\sum_{\ell=1}^{+\infty}\left(S_{-}\right)^{\ell} \mathrm{e}^{-\ell A_{-} w} \Phi_{(0 \mid \ell)}(w) .
\end{aligned}
$$

Rewriting these results for the variable $x=w^{-1}$, making use of Cauchy's theorem (29) for the function $x \Phi_{(0 \mid 0)}(x)$, and expanding for small $x$, we arrive at the large-order predictions $(m \gg 1)$

$$
\begin{aligned}
a_{m}^{(0 \mid 0)} \simeq & -\sum_{k \geq 1} \frac{\left(S_{+}\right)^{k}}{2 \pi \mathrm{i}} \frac{\Gamma\left(m+k \beta_{+}\right)}{\left(k A_{+}\right)^{m+k \beta_{+}}} \sum_{h \geq 0} a_{h}^{(k \mid 0)} \frac{\Gamma\left(m+k \beta_{+}-h\right)}{\Gamma\left(m-k \beta_{+}\right)}\left(k A_{+}\right)^{h}- \\
& -\sum_{k \geq 1} \frac{\left(S_{-}\right)^{k}}{2 \pi \mathrm{i}} \frac{\Gamma\left(m+k \beta_{-}\right)}{\left(k A_{-}\right)^{m+k \beta_{-}}} \sum_{h \geq 0} a_{h}^{(0 \mid k)} \frac{\Gamma\left(m+k \beta_{-}-h\right)}{\Gamma\left(m-k \beta_{-}\right)}\left(k A_{-}\right)^{h} .
\end{aligned}
$$

Given that all the coefficients $a_{m}^{(0 \mid 0)}$ are real, and that the pairs $\beta_{ \pm}, A_{ \pm}$and $a_{h}^{(k \mid 0)}, a_{h}^{(0 \mid k)}$ are complex conjugate, we can easily see that $\frac{S_{+}}{2 \pi \mathrm{i}}$ has to be complex conjugate of $\frac{S_{-}}{2 \pi \mathrm{i}}$, and so the Stokes constants are related by

$$
S_{-}=-\bar{S}_{+}
$$

\footnotetext{
${ }^{8}$ Note that for higher sectors there will naturally be additional singular directions in the Borel plane, associated with different combinations of the two instanton actions $A_{ \pm}$, much in the same way as for the one-parameter transseries the sectors $\Phi_{n}, n \geq 2$ has discontinuities in both the positive and negative real axis.
} 
It will be convenient to define $A_{ \pm}=|A| \mathrm{e}^{ \pm \mathrm{i} \theta_{A}}, \beta_{ \pm}=|\beta| \mathrm{e}^{ \pm \mathrm{i} \theta_{\beta}}=\beta_{R} \pm \mathrm{i} \beta_{I}, S_{ \pm}= \pm|S| \mathrm{e}^{ \pm \mathrm{i} \theta_{S}}$. The leading behaviour of the large-order relations written above is dictated by the sectors $a_{h}^{(1 \mid 0)}$ and $a_{h}^{(0 \mid 1)}$,

$$
a_{m}^{(0 \mid 0)} \simeq-\frac{S_{+}}{2 \pi \mathrm{i}} \frac{\Gamma\left(m+\beta_{+}\right)}{A_{+}^{m+\beta_{+}}} \sum_{h \geq 0} a_{h}^{(1 \mid 0)} \prod_{\ell=1}^{h} \frac{A_{+}}{\left(m+\beta_{+}-\ell\right)}+h . c .+\mathcal{O}\left(2^{-m}\right),
$$

where h.c. stands for the Hermitian conjugate. Unlike the one-parameter case previously studied, in these large-order relations there will always be a dependence on the Stokes constant $S_{+}$. Thus, before proceeding with deeper tests of these relations, we need to numerically determine the Stokes constants. This can be done as follows. Defining

$$
Q_{m}=-\frac{1}{2 \pi \mathrm{i}} \frac{\Gamma\left(m+\beta_{+}\right)}{A_{+}^{m+\beta_{+}}} \sum_{h \geq 0} a_{h}^{(1 \mid 0)} \prod_{\ell=1}^{h} \frac{A_{+}}{\left(m+\beta_{+}-\ell\right)}
$$

one has

$$
a_{m}^{(0 \mid 0)} \simeq S_{+} Q_{m}+\text { h.c. }+\mathcal{O}\left(2^{-m}\right)
$$

If we can determine a resummed value for the $Q_{m} \equiv\left|Q_{m}\right| \mathrm{e}^{\mathrm{i} \theta_{Q}(m)}$, for each $m$, then it will easily follow that

$$
\frac{a_{m+1}^{(0 \mid 0)}}{a_{m}^{(0 \mid 0)}} \simeq \frac{\left|Q_{m+1}\right|}{\left|Q_{m}\right|} \frac{\cos \left(\theta_{Q}(m+1)+\theta_{S}\right)}{\cos \left(\theta_{Q}(m)+\theta_{S}\right)} .
$$

Note that this relation is still a large-order relation, i.e., it is valid for large values of $m$. The argument of the Stokes constant can then be found by rewriting this large-order relation Eq. (56),

$$
\tan \theta_{S}=\frac{g(m) \cos \theta_{Q}(m)-\cos \theta_{Q}(m+1)}{g(m) \sin \theta_{Q}(m)-\sin \theta_{Q}(m+1)}
$$

where

$$
g(m) \equiv \frac{a_{m+1}^{(0 \mid 0)}}{a_{m}^{(0 \mid 0)}} \frac{\left|Q_{m}\right|}{\left|Q_{m+1}\right|} .
$$

To determine the resummed values of $Q_{m}$, first notice that the sum present in (54) is asymptotic for large $m$ :

$$
\eta(m) \equiv \sum_{h \geq 0} a_{h}^{(1 \mid 0)} \prod_{\ell=1}^{h} \frac{A_{+}}{\left(m+\beta_{+}-\ell\right)} \simeq \sum_{k=0}^{+\infty} \frac{\eta_{k}}{m^{k}}
$$


The coefficients $\eta_{k}$ are fully determined by the value of $A_{+}, \beta_{+}$and the coefficients $a_{h}^{(1 \mid 0)}$. The latter were determined from the recurrence relations coming from the original differential equation, up to $h=100$. The above sum can be computed via the Borel-Padé resummation method (see Ref. [33] for more details) $:^{9}$

- We first determine the Borel transform corresponding to the asymptotic sum $\eta(m)$, Eq. (59).

- We approximate this Borel transform by a diagonal Padé approximant of order $N=50$, denoted by $\mathrm{BP}_{50}[\eta]$.

- The resummed series $\mathcal{S} \eta(m)$ is then determined via the usual Laplace transform. along the positive real axis as we want $m \in \mathbb{N}$

$$
\mathcal{S} \eta(m)=\int_{0}^{+\infty} d s \mathrm{e}^{-s m} \mathrm{BP}_{50}[\eta](s)
$$

This was performed for $m=1, \cdots, 100{ }^{10}$

We can finally rewrite the resummed $Q_{m}$ as

$$
\mathcal{S} Q_{m}=-\frac{1}{2 \pi \mathrm{i}} \frac{\Gamma\left(m+\beta_{+}\right)}{A_{+}^{m+\beta_{+}}} \mathcal{S} \eta(m) .
$$

With this result we can determine the argument of the Stokes constant $\theta_{S} \equiv \arg \left(S_{+}\right)$for each value of $m$ via the relation (57), by substituting the resummed value $\mathcal{S} Q_{m}$ for the $Q_{m}$. The result is illustrated in Fig. 6, the phase becomes essentially independent of $m$ and is given by $\theta_{S}=-1.710276$ (the estimated error is of order $10^{-6}$ ).

One can similarly calculate the modulus of the Stokes constant. From Eq. (55) it follows that for large $m$ the modulus of the Stokes constant $|S| \equiv\left|S_{+}\right|$should converge to

$$
|S| \simeq \frac{a_{m}^{(0 \mid 0)}}{2\left|Q_{m}\right| \cos \left(\theta_{S}+\theta_{Q}(m)\right)},
$$

with the $Q_{m}$ replaced by the resummed ones given in Eq. (61). This convergence can be seen in Fig. 7, implying the value $|S|=4.728045$ (with error of order $10^{-6}$ ).

\footnotetext{
${ }^{9}$ This sum could also be approximated by performing an optimal truncation for each value of $m$.

${ }^{10}$ For this sum, the positive real axis is not a Stokes line, and there is no ambiguity associated with the resummation.
} 


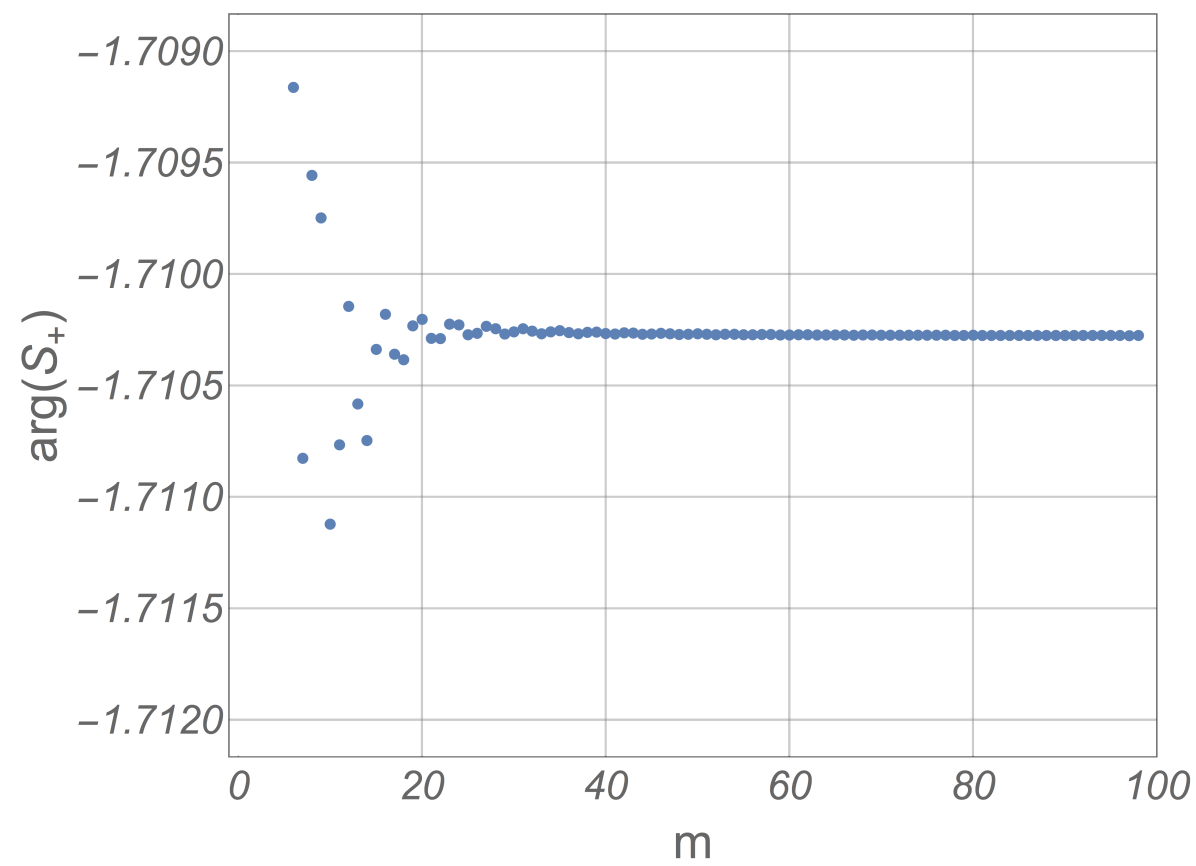

Figure 6: Convergence of the phase of the Stokes constant $\theta_{S} \equiv \arg \left(S_{+}\right)$. We plot 57 for each $m$ using the resummed $Q_{m}$.

This concludes the numerical calculation of the Stokes constant. Using its value we can now check the resurgence large-order relations (53).

Let us define a new quantity by

$$
\Omega(m) \equiv \frac{2 \pi}{|S|} \frac{|A|^{m+\beta_{R}} \mathrm{e}^{-\beta_{I} \theta_{A}}}{m^{\beta_{R}} \Gamma(m)} a_{m}^{(0 \mid 0)} .
$$

Making use of the asymptotic expansion $(59)$, as well as the following large $m$ expansion ${ }^{11}$

$$
\gamma(m) \equiv \frac{\Gamma\left(m+\beta_{+}\right)}{m^{\beta_{R}} \Gamma(m)}=\sum_{k=0}^{+\infty} \frac{\gamma_{k}}{m^{k}}
$$

we can easily find the large order behaviour for $\Omega(m), m \gg 1$ :

$$
\Omega(m) \simeq \mathrm{e}^{\mathrm{i} \Theta(m)} \sum_{k=0}^{+\infty} \frac{c_{k}}{m^{k}}+\text { h.c. }=2 \sum_{k=0}^{+\infty} \frac{\left|c_{k}\right|}{m^{k}} \cos \left(\Theta(m)+\theta_{c}(k)\right) .
$$

\footnotetext{
${ }^{11}$ As an example we present the first three terms in this expansion: $\gamma_{0}=1, \gamma_{1}=\frac{1}{2} \beta_{+}\left(\beta_{+}-1\right)$, and $\gamma_{3}=\frac{1}{24} \beta_{+}\left(\beta_{+}-1\right)\left(\beta_{+}-2\right)\left(3 \beta_{+}-1\right)$.
} 


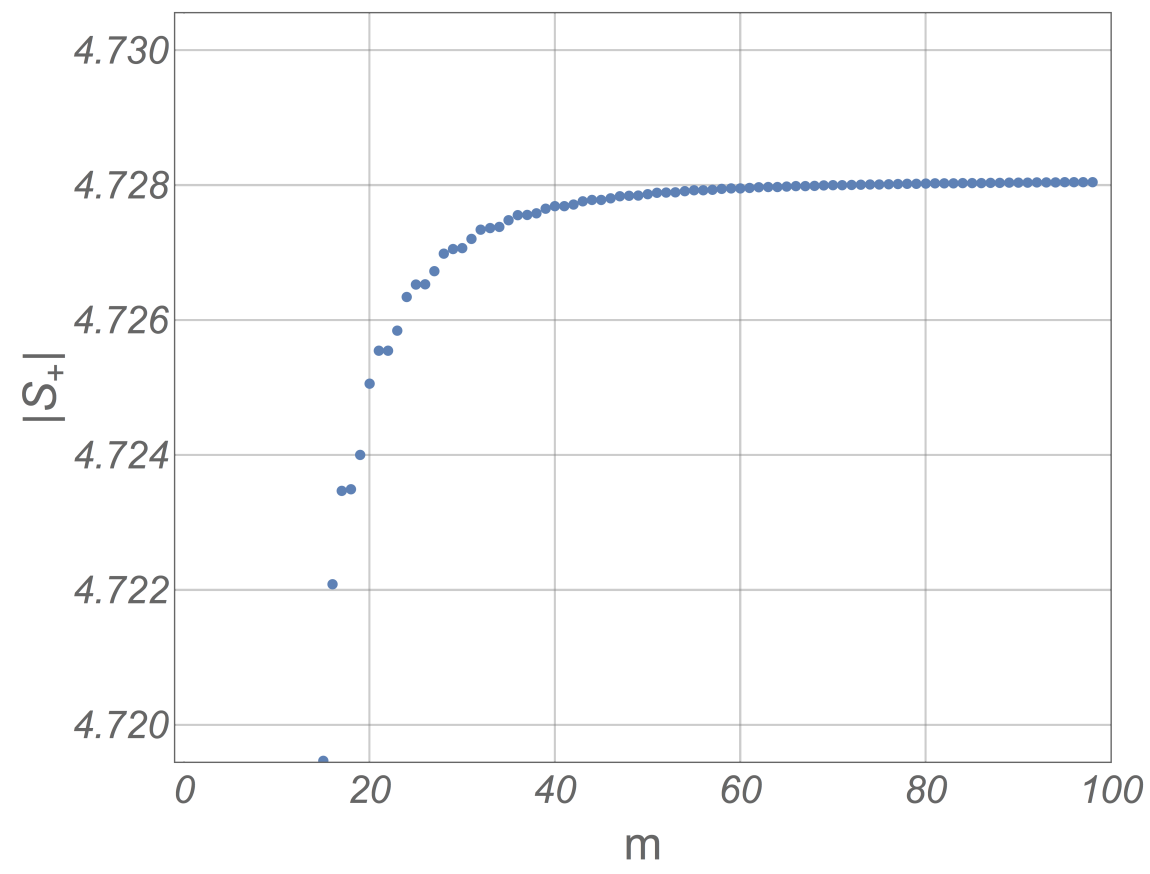

Figure 7: Convergence of the large-order relation Eq. 62 to the modulus of the Stokes constant $|S| \equiv\left|S_{+}\right|$.

The coefficients $c_{k} \equiv\left|c_{k}\right| \mathrm{e}^{\mathrm{i} \theta_{c}(k)}$ appearing in this expression are defined by

$$
\sum_{k \geq 0} c_{k} m^{-k} \equiv \gamma(m) \eta(m)
$$

and the angle $\Theta(m)$ is

$$
\Theta(m) \equiv \frac{\pi}{2}+\theta_{S}-\theta_{A}\left(m+\beta_{R}\right)-\beta_{I} \log |A|
$$

Note that the coefficients $c_{k}$ are known numerically, because both expansions $\eta(m), \gamma(m)$ are known.

Analysing the relation (65) we find that to leading order in $m\left(\right.$ since $\left.c_{0}=1\right)$

$$
\Omega(m) \simeq 2 \cos (\Theta(m))+\mathcal{O}\left(m^{-1}\right)
$$

In Figs. 8 and 9 we can see the convergence of the numerical results to the predicted behaviour for two different ranges of $m$ : for the range of $m<100$ we can see a slow convergence, getting more accurate for higher values of $m$; in the range $500<m<600$ there is already complete consistency between numerical results and predicted behaviour. 


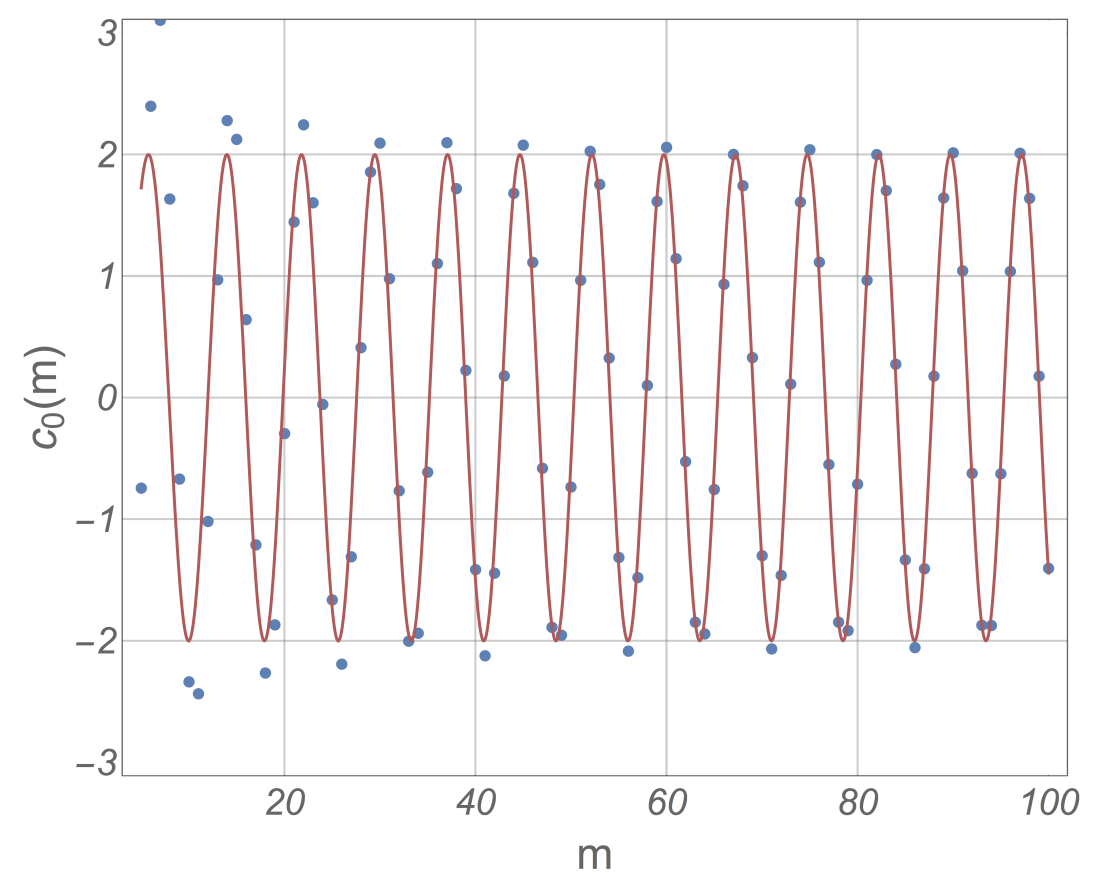

Figure 8: Convergence of the large-order relation 65) to the expected behaviour predicted by the coefficient $c_{0}(m) \equiv 2 \cos (\Theta(m))$.

We can also study the convergence of the large-order relations to a general coefficient $c_{k}$ for some specific $k$ by subtracting the first $k-1$ elements of the series and multiplying the result by $m^{k}$. For example, we can check the convergence to the term $k=2$ in the relation 65 blotting

$$
\left(\Omega(m)-2 \sum_{k=0}^{1} \frac{\left|c_{k}\right|}{m^{k}} \cos \left(\Theta(m)+\theta_{c}(k)\right)\right) m^{2} \simeq 2\left|c_{2}\right| \cos \left(\Theta(m)+\theta_{c}(2)\right)+\mathcal{O}\left(m^{-1}\right) .
$$

This convergence can be seen in Fig. 10: for the range of large $m$ presented, we find consistency between numerical and predicted results. It is important to note that the convergence to higher coefficients $c_{k}$ is highly nontrivial, and is based on the assumption that the transseries is resurgent and that the value of the Stokes constant has been correctly determined. If either of these assumptions had failed, we would not have found convergence of the numerics to higher orders predicted by resurgence.

It is also of importance to point out the slight deviation of the numerical data from 


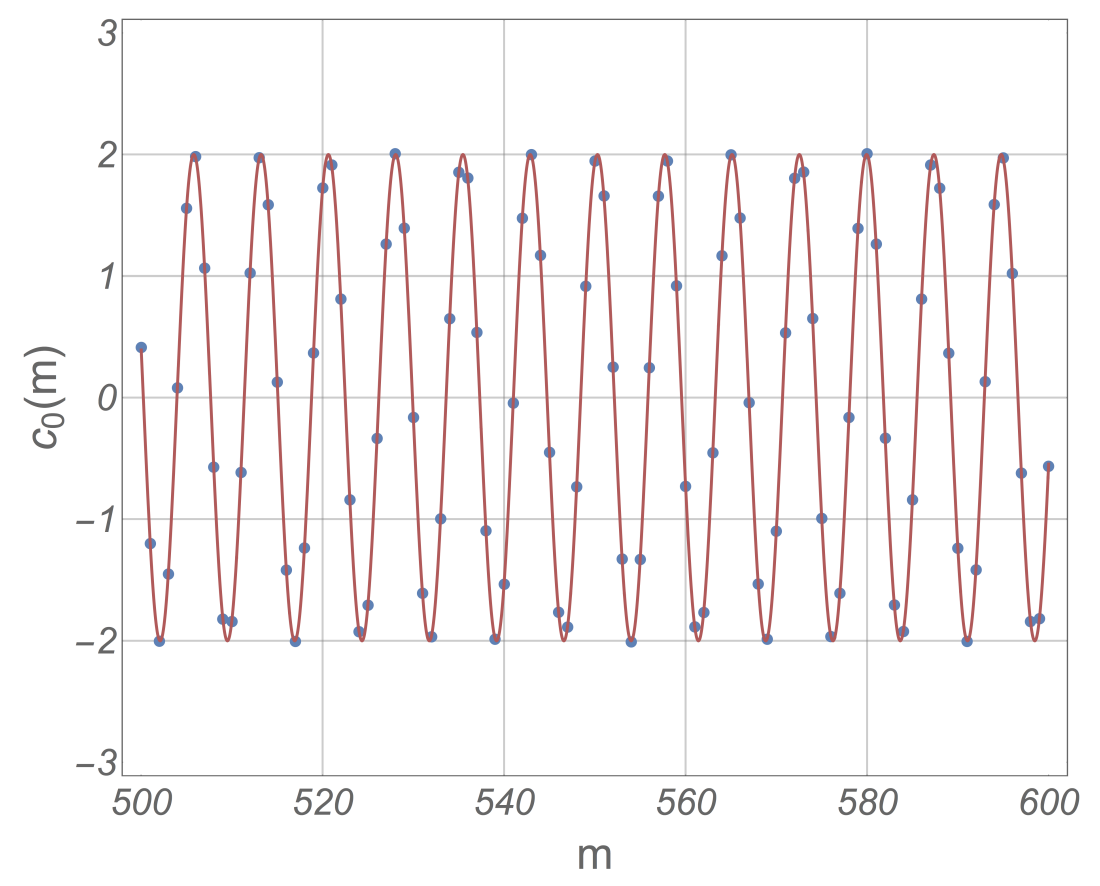

Figure 9: Consistency of the large-order relation 65) with the expected behaviour predicted by the coefficient $c_{0}(m) \equiv 2 \cos (\Theta(m))$ at large $m$.

the predicted values in Fig. 10. The main reason for this is that we have determined (based on the same numerical data) the values of the Stokes constants with an error of $10^{-6}$; this error will eventually cause such a deviation. In order to get more accurate results in the convergence to higher sectors, one would need to determine more coefficients of the sectors $\Phi_{(1 \mid 0)}$ and $\Phi_{(0 \mid 1)}$ and use them to lower the numerical error of the Stokes constant calculation.

Now that we have confirmed the resurgent properties of the perturbative series ${ }^{12}$ we turn to the central question: how to resum our two-parameter transseries (45). We want to resum our transseries for positive real coupling. Because the singularities in the Borel plane are away from this direction, we can perform the integration of the Laplace transform 22 , where now the sectors being resummed are the $\Phi_{(n \mid m)}$. There is no ambiguity involved in

\footnotetext{
${ }^{12}$ The resurgent properties of higher nonperturbative sectors can also be checked, once higher sectors are determined via recursion relations and resummations of the lower sectors are performed.
} 


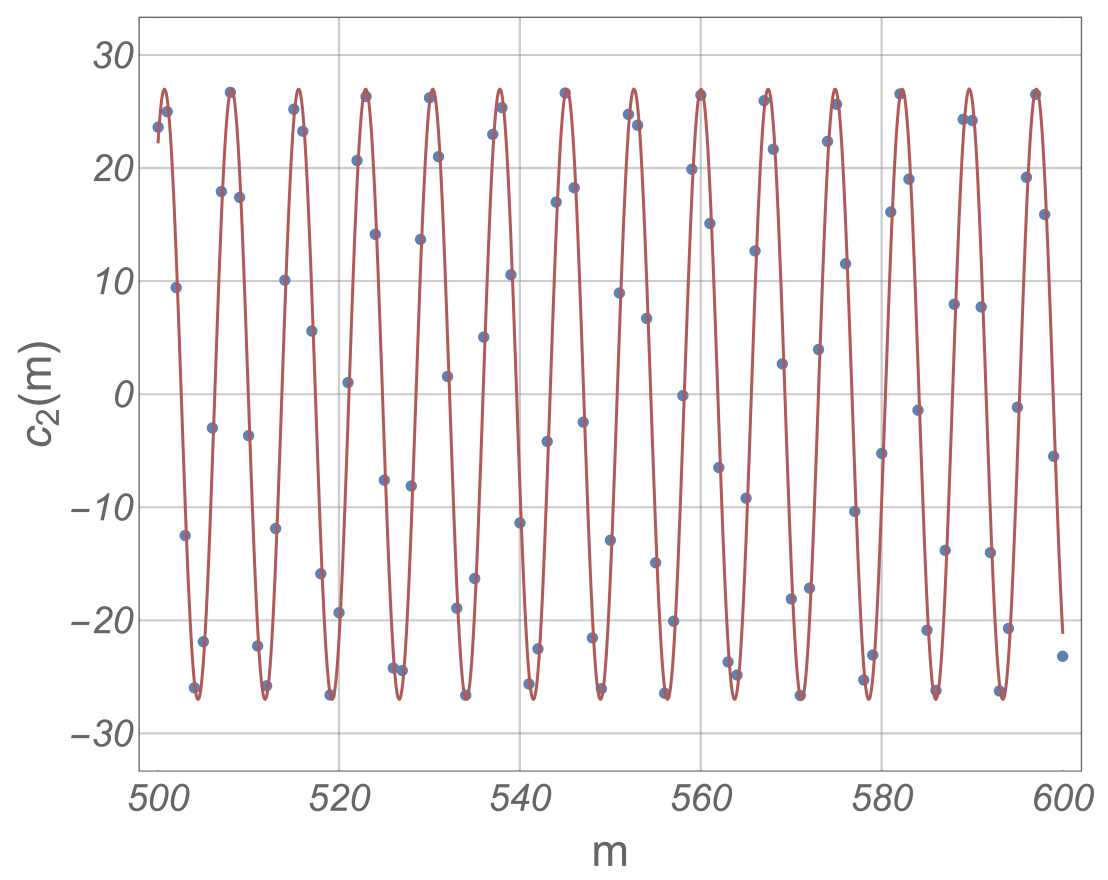

Figure 10: Consistency of the large-order relation Eq. 69 with the expected behaviour predicted by the coefficient $c_{2}(m) \equiv 2\left|c_{2}\right| \cos \left(\Theta(m)+\theta_{c}(2)\right)$ at large $m$.

this calculation, and the resummed transseries (for the positive real line $\theta=0$ ) is given by

$$
\mathcal{S}_{0} f\left(w, \sigma_{ \pm}\right)=\sum_{n, m=0}^{+\infty} \sigma_{+}^{n} \sigma_{-}^{m} \mathrm{e}^{-\left(n A_{+}+m A_{-}\right) w} \mathcal{S}_{0} \Phi_{(n \mid m)}(w)
$$

We could be tempted to set the transseries parameters $\sigma_{ \pm}=0$, which would leave us with only the perturbative series. But the nonperturbative sectors will give us some real exponentially suppressed contributions that we should not neglect as they will play a role in the correct final answer ${ }^{13}$ In fact, this was already seen in other problems of resummation 46, 44. Consequently, we should allow for nonzero $\sigma_{ \pm}$.

For real values of $w$ we expect a real solution, and we know that sectors $\Phi_{(n \mid m)}$ are complex conjugate to $\Phi_{(m \mid m)}$, with the instanton actions also being complex conjugates. Therefore in order to have a real solution we need to have $\sigma_{+}^{n} \sigma_{-}^{m}$ be complex conjugate to

\footnotetext{
${ }^{13}$ If the actions had a negative real part, then one should in fact set the parameters to zero when considering the transseries in the positive real axis, as not to have exponentially enhanced contributions.
} 
$\sigma_{+}^{m} \sigma_{-}^{n}$ for any $m, n$. In particular putting $m=0, n=1$ we find

$$
\sigma_{+}=\bar{\sigma}_{-} \equiv \sigma
$$

Writing the first few terms of the resummed transseries (70), we have $\left(A_{ \pm}=A_{R} \pm \mathrm{i} A_{I}\right)$

$$
\begin{aligned}
\mathcal{S}_{0} f(w, \sigma)= & \sum_{n=0}^{+\infty} \mathrm{e}^{-n A_{R} w} \sum_{m=0}^{n} \sigma^{n-m} \bar{\sigma}^{m} \mathrm{e}^{-\mathrm{i}(n-2 m) A_{I} w} \mathcal{S}_{0} \Phi_{(n \mid m)}(w) \\
= & \mathcal{S}_{0} \Phi_{(0 \mid 0)}(w)+\mathrm{e}^{-A_{R} w} 2 \operatorname{Re}\left(\sigma \mathrm{e}^{-\mathrm{i} A_{I} w} \mathcal{S}_{0} \Phi_{(1 \mid 0)}(w)\right)+ \\
& +\mathrm{e}^{-2 A_{R} w}\left[2 \operatorname{Re}\left(\sigma^{2} \mathrm{e}^{-2 \mathrm{i} A_{I} w} \mathcal{S}_{0} \Phi_{(2 \mid 0)}(w)\right)+|\sigma|^{2} \mathcal{S}_{0} \Phi_{(1 \mid 1)}(w)\right]+\mathcal{O}\left(\mathrm{e}^{-3 A_{R} w}\right) .
\end{aligned}
$$

Note that the complex number $\sigma$ is not determined by the above analysis. This freedom corresponds exactly to the two integration constants expected for a solution of a second-order ordinary differential equation and can be fixed by imposing suitable initial conditions.

\section{$7 \quad$ Summary and conclusions}

The equations of hydrodynamics constitute a physically well-motivated coarse grained description of a wide range of phenomena. It has recently become clear that they provide a new area of application for resurgence ideas. We have tried to describe a mature version of these ideas in the context of MIS theory, which provides the simplest example of an infinite hydrodynamic series. This series is divergent in a way which encodes information about the nonhydrodynamic mode present in MIS theory.

The main point of this paper was to apply these methods to a hydrodynamic model which aims to describe a richer spectrum of nonhydrodynamic modes, inspired by what is seen in $\mathcal{N}=4 \mathrm{SYM}$. We have shown in some detail that also in this theory the hydrodynamic solution is the leading term in a transseries expansion. These results confirm general expectations concerning the nature of gradient expansions [69]. They also provide an interesting example of resurgent transseries, where the nonperturbative sectors not only have the expected exponentially suppressed behaviour at late times $(w \gg 1)$, but also an 
oscillatory one. This oscillatory behaviour will become more pronounced in early times, when these sectors are no longer suppressed - even though there are no ambiguities in this problem, the full transseries is still needed to account for this behaviour. From the point of view of resurgence theory, this oscillatory behaviour also brought novel features. Because the large-order relations cannot be disentangled from the Stokes constants, and one cannot use normal convergence acceleration methods due to the oscillations, we needed to introduce a Borel-Padé resummation of the first nonperturbative sectors to accurately determine both modulus and argument of the Stokes constant. This then allowed us to check the large-order relations with high accuracy.

From a physical perspective, one would like to understand cases where the series expansion is generated directly from some underlying microscopic quantum theory, such as strongly coupled $\mathcal{N}=4 \mathrm{SYM}$. In this case there is an infinite sequence of nonhydrodynamic modes corresponding to the black brane quasinormal modes [70,68]. To include more than a single pair of complex conjugate quasinormal modes would involve multiparameter transseries, with each quasinormal mode defining (in principle) a separate Stokes line.

In practice one would first aim at understanding the effects of the leading modes those with the longest relaxation times. In the case of boost-invariant flow in $\mathcal{N}=4 \mathrm{SYM}$, the hydrodynamic series has already been computed to high order, and the calculation of at least a few terms of the 1-instanton sector series is feasible. In conjunction with the methods developed in the study presented here, this opens up the possibility of at least checking consistency with resurgence in this very important case.

Acknowledgments: we would like to thank Michał Heller and Ricardo Schiappa for useful comments on the manuscript. I.A. was supported by the National Science Centre grant 2012/06/A/ST2/00396. M.S. was supported by the National Science Centre grant 2012/07/B/ST2/03794. 


\section{References}

[1] R. Baier, P. Romatschke, D. T. Son, A. O. Starinets, and M. A. Stephanov, "Relativistic viscous hydrodynamics, conformal invariance, and holography," JHEP 0804 (2008) 100, arXiv:0712.2451 [hep-th].

[2] P. Romatschke, "New Developments in Relativistic Viscous Hydrodynamics," Int. J. Mod. Phys. E19 (2010) 1-53, arXiv:0902.3663 [hep-ph].

[3] E. Shuryak, "Heavy Ion Collisions: Achievements and Challenges," arXiv:1412.8393 $[$ hep-ph].

[4] M. P. Heller, R. A. Janik, and P. Witaszczyk, "Hydrodynamic Gradient Expansion in Gauge Theory Plasmas," Phys.Rev.Lett. 110 (2013) no. 21, 211602 , arXiv:1302.0697 [hep-th].

[5] M. P. Heller and M. Spalinski, "Hydrodynamics Beyond the Gradient Expansion: Resurgence and Resummation," Phys. Rev. Lett. 115 (2015) no. 7, 072501, arXiv:1503.07514 [hep-th].

[6] J. Bjorken, "Highly Relativistic Nucleus-Nucleus Collisions: The Central Rapidity Region," Phys.Rev. D27 (1983) 140-151.

[7] R. A. Janik and R. B. Peschanski, "Asymptotic perfect fluid dynamics as a consequence of Ads/CFT," Phys. Rev. D73 (2006) 045013, arXiv:hep-th/0512162 [hep-th].

[8] I. Muller, "Zum Paradoxon der Warmeleitungstheorie," Z.Phys. 198 (1967) 329-344.

[9] W. Israel and J. Stewart, "Transient relativistic thermodynamics and kinetic theory," Annals Phys. 118 (1979) 341-372.

[10] C. M. Bender and T. T. Wu, "Anharmonic Oscillator," Phys. Rev. 184 (1969) 1231. 
[11] C. M. Bender and T. Wu, "Anharmonic Oscillator 2: A Study of Perturbation Theory in Large Order," Phys. Rev. D7 (1973) 1620.

[12] E. Bogomolny, "Calculation of Instanton-Anti-Instanton Contributions in Quantum Mechanics," Phys. Lett. B91 (1980) 431.

[13] J. Zinn-Justin, "Multi - Instanton Contributions in Quantum Mechanics," Nucl.Phys. B192 (1981) 125-140.

[14] J. Zinn-Justin, "Multi - Instanton Contributions in Quantum Mechanics. 2.," Nucl.Phys. B218 (1983) 333-348.

[15] J. Zinn-Justin, "Instantons in Quantum Mechanics: Numerical Evidence for a Conjecture," J. Math. Phys. 25 (1984) 549.

[16] J. Zinn-Justin and U. D. Jentschura, "Multi-Instantons and Exact Results I: Conjectures, WKB Expansions, and Instanton Interactions," Annals Phys. 313 (2004) 197, arXiv:quant-ph/0501136 [quant-ph].

[17] J. Zinn-Justin and U. D. Jentschura, "Multi-Instantons and Exact Results II: Specific Cases, Higher-Order Effects, and Numerical Calculations," Annals Phys. 313 (2004) 269, arXiv:quant-ph/0501137 [quant-ph].

[18] Z. Ambroziński and J. Wosiek, "Resummation of Not Summable Series," arXiv:1210.3554 [quant-ph].

[19] G. V. Dunne and M. Ünsal, "Generating nonperturbative physics from perturbation theory," Phys.Rev. D89 (2014) no. 4, 041701, arXiv:1306.4405 [hep-th].

[20] G. V. Dunne and M. Unsal, "Uniform WKB, Multi-instantons, and Resurgent Trans-Series," Phys.Rev. D89 (2014) no. 10, 105009, arXiv:1401.5202 [hep-th]. 
[21] P. C. Argyres and M. Ünsal, "The Semi-Classical Expansion and Resurgence in Gauge Theories: New Perturbative, Instanton, Bion, and Renormalon Effects," JHEP 1208 (2012) 063, arXiv:1206.1890 [hep-th].

[22] G. V. Dunne and M. Ünsal, "Resurgence and Trans-Series in Quantum Field Theory: The $\mathbb{C P}^{N-1}$ Model," JHEP 1211 (2012) 170, arXiv:1210.2423 [hep-th].

[23] I. Aniceto and R. Schiappa, "Nonperturbative Ambiguities and the Reality of Resurgent Transseries," Commun.Math.Phys. 335 (2015) no. 1, 183-245, arXiv:1308.1115 [hep-th].

[24] I. Aniceto, "The Resurgence of the Cusp Anomalous Dimension," To appear in J. Phys. A (2015), arXiv:1506.03388 [hep-th].

[25] D. Dorigoni and Y. Hatsuda, "Resurgence of the Cusp Anomalous Dimension," JHEP 09 (2015) 138, arXiv:1506.03763 [hep-th].

[26] B. Candelpergher, J. Nosmas, and F. Pham, "Premiers Pas en Calcul Étranger," Ann. Inst. Fourier 43 (1993) 201.

[27] E. Delabaere, "Effective Resummation Methods for an Implicit Resurgent Function," arXiv:math-ph/0602026 [math-ph].

[28] T. Seara and D. Sauzin, "Resumació de Borel i Teoria de la Ressurgència," Butl. Soc. Catalana Mat. 18 (2003) 131.

[29] D. Sauzin, "Introduction to 1-summability and resurgence," arXiv:1405.0356 [math.DS].

[30] D. Dorigoni, "An Introduction to Resurgence, Trans-Series and Alien Calculus," arXiv:1411.3585 [hep-th].

[31] G. A. Edgar, "Transseries for Beginners," Real Anal. Exchange 35 (2009) 253, arXiv:0801.4877 [math.RA], 
[32] M. Mariño, "Nonperturbative Effects and Nonperturbative Definitions in Matrix Models and Topological Strings," JHEP 0812 (2008) 114, arXiv:0805.3033 [hep-th].

[33] I. Aniceto, R. Schiappa, and M. Vonk, "The Resurgence of Instantons in String Theory," Commun. Num. Theor. Phys. 6 (2012) 339, arXiv:1106.5922 [hep-th].

[34] M. Mariño, "Lectures on non-perturbative effects in large $N$ gauge theories, matrix models and strings," Fortsch.Phys. 62 (2014) 455-540, arXiv:1206.6272 [hep-th].

[35] I. Aniceto, G. Başar, and R. Schiappa, "A Primer on the Resurgent Asymptotics of Perturbative Expansions," upcoming (2015) .

[36] R. Couso-Santamaría, J. D. Edelstein, R. Schiappa, and M. Vonk, "Resurgent Transseries and the Holomorphic Anomaly," Annales Henri Poincaré, in press (2013) , arXiv:1308.1695 [hep-th].

[37] R. Couso-Santamaría, J. D. Edelstein, R. Schiappa, and M. Vonk, "Resurgent Transseries and the Holomorphic Anomaly: Nonperturbative Closed Strings in Local $\mathbb{C P}^{2}$," Commun.Math.Phys. 338 (2015) no. 1, 285-346, arXiv:1407.4821 [hep-th].

[38] Y. Hatsuda and K. Okuyama, "Resummations and Non-Perturbative Corrections," arXiv:1505.07460 [hep-th].

[39] R. Couso-Santamaria, "Universality of the topological string at large radius and NS-brane resurgence," arXiv:1507.04013 [hep-th].

[40] G. Başar, G. V. Dunne, and M. Ünsal, "Resurgence theory, ghost-instantons, and analytic continuation of path integrals," JHEP 1310 (2013) 041, arXiv:1308.1108 [hep-th].

[41] A. Behtash, E. Poppitz, T. Sulejmanpasic, and M. Ünsal, "The curious incident of multi-instantons and the necessity of Lefschetz thimbles," JHEP 11 (2015) 175. arXiv:1507.04063 [hep-th]. 
[42] A. Cherman, D. Dorigoni, and M. Unsal, "Decoding perturbation theory using resurgence: Stokes phenomena, new saddle points and Lefschetz thimbles," arXiv:1403.1277 [hep-th].

[43] G. V. Dunne and M. Ünsal, "Resurgence and Dynamics of $\mathrm{O}(\mathrm{N})$ and Grassmannian Sigma Models," arXiv:1505.07803 [hep-th].

[44] R. Couso-Santamaría, R. Schiappa, and R. Vaz, "Finite N from Resurgent Large N," Annals Phys. 356 (2015) 1-28, arXiv:1501.01007 [hep-th].

[45] A. Cherman, P. Koroteev, and M. Ünsal, "Resurgence and Holomorphy: From Weak to Strong Coupling," J. Math. Phys. 56 (2015) no. 5, 053505, arXiv:1410.0388 $[$ hep-th].

[46] A. Grassi, M. Marino, and S. Zakany, "Resumming the string perturbation series," JHEP 1505 (2015) 038, arXiv:1405.4214 [hep-th].

[47] I. Aniceto, J. G. Russo, and R. Schiappa, "Resurgent Analysis of Localizable Observables in Supersymmetric Gauge Theories," JHEP 1503 (2015) 172, arXiv:1410.5834 [hep-th].

[48] G. Başar and G. V. Dunne, "Resurgence and the Nekrasov-Shatashvili limit: connecting weak and strong coupling in the Mathieu and Lamé systems," JHEP 1502 (2015) 160, arXiv: 1501.05671 [hep-th].

[49] M. P. Heller, R. A. Janik, M. Spaliński, and P. Witaszczyk, "Coupling hydrodynamics to nonequilibrium degrees of freedom in strongly interacting quark-gluon plasma," Phys.Rev.Lett. 113 (2014) no. 26, 261601, arXiv:1409.5087 [hep-th].

[50] J. Bhattacharya, S. Bhattacharyya, S. Minwalla, and A. Yarom, "A Theory of first order dissipative superfluid dynamics," JHEP 05 (2014) 147, arXiv:1105.3733 $[$ hep-th]. 
[51] R. Loganayagam, "Entropy Current in Conformal Hydrodynamics," JHEP 0805 (2008) 087, arXiv:0801.3701 [hep-th].

[52] L. D. Landau and E. M. Lifshitz, Fluid Mechanics, Second Edition: Volume 6 (Course of Theoretical Physics). 1987.

[53] W. A. Hiscock and L. Lindblom, "Generic instabilities in first-order dissipative relativistic fluid theories," Phys.Rev. D31 (1985) 725-733.

[54] P. Kostädt and M. Liu, "Causality and stability of the relativistic diffusion equation," Phys.Rev. D62 (2000) 023003, arXiv: cond-mat/0010276.

[55] R. P. Geroch, "Relativistic theories of dissipative fluids," J.Math.Phys. 36 (1995) 4226.

[56] R. P. Geroch, "On hyperbolic 'theories' of relativistic dissipative fluids," arXiv:gr-qc/0103112 [gr-qc].

[57] M. P. Heller and R. A. Janik, "Viscous hydrodynamics relaxation time from AdS/CFT," Phys. Rev. D76 (2007) 025027, arXiv:hep-th/0703243 [HEP-TH].

[58] S. Bhattacharyya, V. E. Hubeny, S. Minwalla, and M. Rangamani, "Nonlinear Fluid Dynamics from Gravity," JHEP 0802 (2008) 045, arXiv:0712.2456 [hep-th].

[59] M. Luzum and P. Romatschke, "Conformal Relativistic Viscous Hydrodynamics: Applications to RHIC results at s(NN)**(1/2) = 200-GeV," Phys.Rev. C78 (2008) 034915, arXiv:0804.4015 [nucl-th].

[60] M. P. Heller, R. A. Janik, and P. Witaszczyk, "The characteristics of thermalization of boost-invariant plasma from holography," Phys.Rev.Lett. 108 (2012) 201602, arXiv:1103.3452 [hep-th].

[61] J. C. Collins and D. E. Soper, "Large Order Expansion in Perturbation Theory," Annals Phys. 112 (1978) 209-234. 
[62] M. Mariño, R. Schiappa, and M. Weiss, "Nonperturbative Effects and the Large-Order Behavior of Matrix Models and Topological Strings," Commun. Num. Theor. Phys. 2 (2008) 349, arXiv:0711.1954 [hep-th].

[63] S. Garoufalidis, A. Its, A. Kapaev, and M. Mariño, "Asymptotics of the Instantons of Painlevé I," Int. Math. Res. Notices 2012 (2012) 561, arXiv:1002.3634 [math.CA]

[64] R. Schiappa and R. Vaz, "The Resurgence of Instantons: Multi-Cut Stokes Phases and the Painlevé II Equation," Commun.Math.Phys. 330 (2014) 655-721, arXiv:1302.5138 [hep-th].

[65] G. Başar and G. V. Dunne, "Hydrodynamics, resurgence and trans-asymptotics," arXiv:1509.05046 [hep-th].

[66] P. M. Chesler and L. G. Yaffe, "Boost invariant flow, black hole formation, and far-from-equilibrium dynamics in $\mathrm{N}=4$ supersymmetric Yang-Mills theory," Phys.Rev. D82 (2010) 026006, arXiv:0906.4426 [hep-th].

[67] J. Jankowski, G. Plewa, and M. Spalinski, "Statistics of thermalization in Bjorken Flow," JHEP 12 (2014) 105, arXiv:1411.1969 [hep-th].

[68] A. Nunez and A. O. Starinets, "AdS / CFT correspondence, quasinormal modes, and thermal correlators in N=4 SYM," Phys. Rev. D67 (2003) 124013, arXiv:hep-th/0302026 [hep-th].

[69] G. V. Dunne, "Resurgence and Trans-series in Quantum Theories," Lectures given at the Schladming Winter School 2015.

[70] P. K. Kovtun and A. O. Starinets, "Quasinormal modes and holography," Phys.Rev. D72 (2005) 086009, arXiv:hep-th/0506184 [hep-th]. 